\title{
Effect of Couple Stress Fluid in an Irregular Couette Flow Channel: An Analytical Approach
}

\author{
Uma Munivenkatappa 1(D), Dinesh Pobbathy Aswathanarayana 1(i), Avula Sreevallabha Reddy 1(D), \\ Suresh Babu Ramakrishna ${ }^{1, *(D)}$
}

1 Department of Mathematics, M S Ramaiah Institute of Technology, Bangalore-54, India; umashivaram@gmail.com (U.M.); dineshpa@msrit.edu (D.P.A.); sreensiri@gmail.com (A.S.R.); sureshbabu_r80@yahoo.co.in (S.B.R.);

* Correspondence: sureshbabur_r80@yahoo.co.in (S.B.R.);

Scopus Author ID 57199323358

Received: 9.08.2021; Revised: 20.09.2021; Accepted: 23.09.2021; Published: 16.10.2021

\begin{abstract}
The work embodied in this paper presents the combined effects of Soret, chemical reaction, and Dufour on Couette flow in an irregular channel for dusty viscoelastic couple stress fluid. The behavior of the boundary layer is studied with the help of Brinkman-Forchheimer's extended Darcy model as a momentum equation for the unsteady, incompressible dusty viscoelastic fluid. The heat transfers are considered due to the radiation absorption parameter and Dufour effect, the mass transfer influenced by the chemical reaction, and the Soret effect. The boundary conditions of the problem and leading equations of the physical problem are solved by a similarity transformation, and the consequent ordinary linear differential equations are solved by the perturbation method. The obtained results are shown graphically. The computational results show a good agreement between our values and a particular case of the earlier work.
\end{abstract}

Keywords: couple stress; Couette flow; irregular channel; MHD; perturbation technique.

(C) 2021 by the authors. This article is an open-access article distributed under the terms and conditions of the Creative Commons Attribution (CC BY) license (https://creativecommons.org/licenses/by/4.0/).

\section{Nomenclature:}

\begin{tabular}{l|l|l|l}
$S_{r}$ & Soret number & $F$ & Thermal radiation parameter \\
\hline$B_{i}$ & Biot number & $E$ & Viscoelastic parameter \\
\hline$G_{r}$ & Thermal Grashof number & $W$ & Couple stress parameter \\
\hline$G_{c}$ & Solutal Grashof number & $m$ & Magnetic field parameter \\
\hline$K_{r}$ & Chemical reaction parameter & $W$ & Relaxation time parameter for dust particles \\
\hline$P_{r}$ & Prandtl number & $M^{2}$ & Hartmann number \\
\hline$\alpha_{T}$ & Heat absorption parameter & $\mathrm{p}$ & Particle \\
\hline$\alpha_{c}$ & Radiation absorption parameter & $\mathrm{f}$ & Fluid \\
\hline$u_{p}$ & Velocity of the moving plate & $K$ & Permeability coefficient of the porous medium \\
\hline$\delta_{n}$ & Mass concentration of dust particles & $\mu$ & Dynamic viscosity \\
\hline$h_{f}$ & Heat transfer coefficient & $v$ & Kinematic viscosity
\end{tabular}

\section{Introduction}

The Couette flow is defined as the laminar flow of a viscous fluid in the space between the two parallel plates, one of which moves relative to the other. The Couette flow of Newtonian or non-Newtonian fluids has gained significance due to its practical and industrial 
applications like power engineering, food preservation technique, and petroleum production. The Couette flow of viscoelastic fluids has various applications in the field, such as airplanes, spacecraft, tire and cushion making, plastic engineering equipment, etc. The flow of viscoelastic fluid through parallel plates and its heat and mass transfer behaviors are helpful to the study of the nature of boundary layer problems. The study of boundary layer theory for a mixed convection flow through a perpendicular cylinder surrounded by viscous fluids has acknowledged significant theoretical and practical attention. The convection process occurs in various technical and industrial circumstances like electronic devices, nuclear reactors, etc. Therefore, Couette flow plays a vital role in the fields of engineering application. Many of the authors have done extensive work to be acquainted with heat and mass transfer behavior for regular geometries. But in practical situations, it is required to analyze complex geometries where we come across irregular channels, wavy tubes, etc. Later on, a number of researchers have done enormous work on complex geometries even because of their practical applications. In this regard, some authors have contributed to fluid flows over a wavy wall. The laminar Couette flows containing the mass transfer process have applications related to chemical engineering. Some of the applications are in the coating process, solid-liquid extraction. Some of the biochemical applications are in dialyzers, oxygenators, etc.

To study the mechanism of rheologically complex fluids like colloidal fluids, liquid crystals, polymeric suspensions, human blood, etc., it is necessary to study the couple stress behavior, with which we can understand its nature easily. The couple stress fluid can be treated as a special type of non-Newtonian fluid. The couple stress theory projected by stokes says the rotational field in terms of the velocity field for setting up the stress and strain rate relationship. To know the behavior of non-Newtonian fluids, different models are proposed. Among these, the couple stress fluids have different features, namely occurrence of couple stress, body couples, and non-symmetric stress tensor. This type of fluids can be used to describe different kinds of lubricants, blood suspension fluids, etc. The couple stress fluid has applications in the process, such as solidifying liquid crystals, colloidal solutions, chilling the metallic plate in a bath, and extruding polymer fluids. The couple stress model can be applied to biomechanical problems, hence in view of these applications, the Couette flow of couple stress fluid is considered in this thesis. Walter's [1] studied non-Newtonian fluid and its effect on elastic, viscous liquids whose shear rates are the general linear equations of state. The phenomenon of Couette flow with its heat \& mass transfer characteristics has a variety of applications in the field, such as purification of crude oil, polymer technology, and petroleum industry. In view of this, the author Attia [2] analyzed the heat transfer of unsteady Couette flow between two infinite horizontal plates which are non-conducting with dust particles. In view of the applications stated earlier, Mekheimer et al. [3] analyzed the effect of applied magnetic field for couple stress fluid on peristaltic flow. The authors Ashmawy [4] studied the Couette flow of an unsteady, incompressible, isothermal micropolar fluid over an infinite parallel plate by adapting Laplace transform method. The authors [5, 6, 7, 8, 9] contributed their study towards Couette flow through an irregular channel.

In view of the applications of dusty particles like fluid dust separators, design of filters, exhaust nozzles, and rocket engines, the authors Gireesha et al. [10] discussed the heat transfer of a dusty fluid for three dimensional Couette flow analytically. In view of the applications stated earlier that is in the nuclear reactors, magnetohydrodynamic generators, geothermal energy extractions, the authors Harish babu et al. [11] considered heat and mass transfer characteristics of a micro-polar fluid flow with variable permeability and radiation absorption 
through vertical moving plate embedded with porous media. Devika et al. [12] have done analytical work to know the mutual effects of heat source and chemical reaction for incompressible viscous 2-dimensional free convection magnetohydrodynamic flow through a long wavy wall in a porous media using regular perturbation technique by applying suitable boundary conditions. The authors Sheikholeslami et al. [13] studied the heat transfer of a micro-polar fluid analytically using the perturbation method between permeable walls. The results are compared numerically using the $4^{\text {th }}$ order Runge-Kutta method. Ahmad et al. [14] studied three-dimensional Couette flow analytically and numerically for a Newtonian fluid that is electrically conducting and incompressible through two horizontal flat plates bounded by porous medium and analyzed its hydromagnetic effects using a porous medium perturbation and electrical network schemes. Venkateswarulu et al. [15] considered a fluid flow through parallel stationary plates for an electrically conducting in Viscoelastic compressible fluid, accompanied by the heat source and chemical reaction. Job et al. [16] deliberated the Couette flow of Newtonian, incompressible fluid by applying transverse uniform magnetic field under the impact of thermal radiation and exponentially decaying pressure gradient, to solve the coupled nonlinear partial differential equation finite element method has been used and finally, the system of algebraic equations are solved using iterative methods. Uma et al. [17] analyzed the Couette flow by taking the effects of Forchheimer, chemical, and radiation for unsteady dusty viscoelastic fluid through a wavy channel using an analytical method. Due to some of the industrial applications such as oil industries and geothermal operations, Shankar et al. [18] have done a numerical study to know the stability of plane porous Couette flow. Levent Aydinbakar et al. [19] dedicated their work to analyzing the computations by varying the Reynolds number, reference frame, and rotation speed of the cylinder for a Taylor Couette flow.

Gnaneswara reddy et al. [20] attempted to find the hydromagnetic peristaltic motion of an incompressible, electrically conducting couple stress fluid in a porous media through an inclined uneven channel and presented the flow of heat and mass characteristics. The authors Hayat et al. [21] analyzed the squeezing flow of the 2D, incompressible, magnetohydrodynamic, couple stress flow of nanofluid through two parallel plates analytically. Babu et al. [22] analyzed the effects of varying fluid properties on the oscillatory movement of a couple of stress fluids on mixed convection through a vertical plate. Owing to the applications of the Soret effect to the field of geophysics, petroleum reservoir, and groundwater, Malashetty et al. [23] published an analytical work on double-diffusive convection of linear and nonlinear flows in the presence of the Soret effect for a couple of stress fluids. Muhammad Sohail et al. [24] explored the entropy examination of couple stress fluid through a stressed, nonlinear surface and found heat and mass transfer effects for different thermophysical parameters. Ali et al. [25] have done numerical work to know the effects of heat transfer on a 3D flow of a couple of stress fluids with convective boundary conditions. Fan et al. [26, 27, 28, 29] analyzed a study related to modified couple stress. An error analysis has been done by young et al. [30] and corrected the model for different beam models constructed on modified couple stress theory and verified the results using finite element analysis. Owing to the immense applications of nanofluids in the process of heat transfer, the authors Sami et al. [31, 32] have done remarkable work in the field of nano biomaterials with the effects of magnetized couple stress.

The authors Muhammad et al. [33] have done comparative work with respect to the Couette flow of couple stress fluid with an iterative method and homotopy method. Owing to the applications in pharmaceutical, power generation, etc., Rahman et al. [34] studied the 
couple stress fluid flow of Couette-Poiseuille, taking the effects of temperature and magnetic field. Due to the enormous applications of couple stress fluids, the authors Muhammad Arif et al. [35] contributed a comparative study towards the generalized Couette flow of couple stress fluid using fractional derivatives. Farhad et al. [36] have investigated the performance of engine oil by adding molybdenum disulfide nanoparticle Couette flow by introducing the couple stress and found that there is an enhancement in the heat transfer and reduction in the mass transfer due to the addition of nanoparticles. Muhammad Arif et al. [37] have done analytical work to know the nature of nanofluid, made of base fluid blood and gold nanoparticles Couette flow of couple stress fluid using Laplace and finite Fourier sine transform methods.

In the present model, the study's novelty is to know the analytical behavior of unsteady dusty viscoelastic fluids with the effects of radiation and couple stress through an irregular channel. To solve the differential equations perturbation technique is employed.

\section{Mathematical Formulation}

Consider an electrically conducting, dusty viscoelastic or Walters fluid Couette flow of two dimensional, unsteady, incompressible fluids in a wavy wall or an irregular channel where the temperature of the fluid reduces near to the channel by convection and with different mass diffusion saturated with porous medium. The $\mathrm{X}$-axis is set to motion which is towards the direction of the irregular channel, and upright to the channel direction, there is Y-axis. The magnetic field of strength $B o$ and the buoyancy effects are applied transversely to the flow field. The conventions made here are low Reynolds number, because of which the applied magnetic field in the system and the hall effects are ignored.

The heat equation comprises of some of the effects like thermal diffusion, heat absorption, radiative heat flux, viscous dissipation, joule effect, and radiation absorption, which are used to transport heat, whereas the concentration equation has the effects such as thermal diffusion and the chemical reaction parameter. The irregular channel sustains the convective boundary settings and follows Newton's law of cooling.

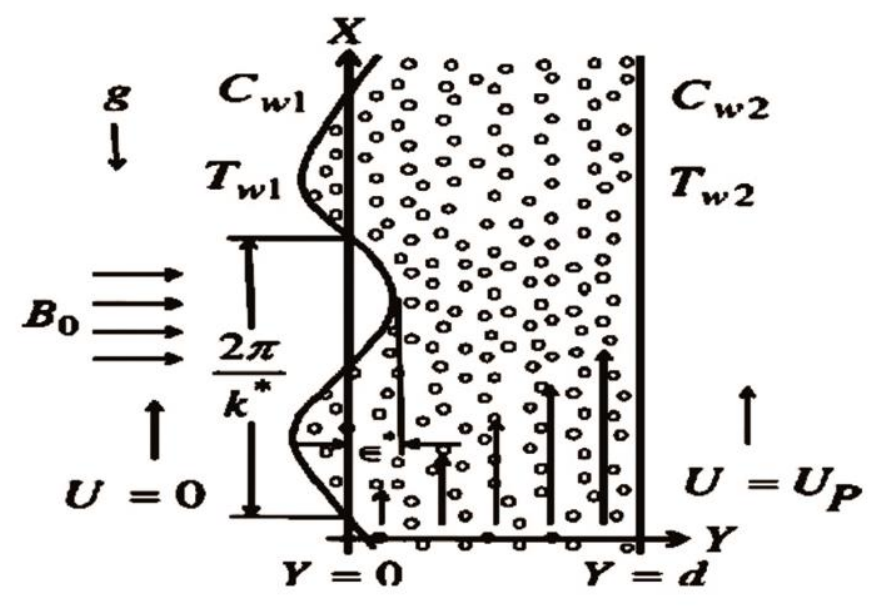

Figure 1. Physical structure of the model.

In Figure $1 T_{2}$ and $T_{1}$ are the temperature of the flat wall and the irregular wall are similar $C_{2}$ and $C_{1}$ are the concentration of the flat wall and irregular wall. $T_{W_{1}}$ and $C_{W_{1}}$ are convective boundary settings for temperature and concentration of the irregular wall. The temperature and concentration of the flat wall are larger than that of temperature and 
concentration of the wavy wall. The convective boundary conditions are given by $-k \frac{\partial T}{\partial Y}=h_{f}\left(T_{1}-T+\left(T_{2}-T_{1}\right) A t^{*}\right), C_{W_{1}}=C_{1}+\left(C_{2}-C_{1}\right) A t^{*}$ for an irregular wall and $-k \frac{\partial T}{\partial Y}=h_{f}\left(T_{2}-T+\left(T_{2}-T_{1}\right) A t^{*}\right), C_{W_{2}}=C_{2}+\left(C_{2}-C_{1}\right) A t^{*}$ for a flat wall. Here, thermal conductivity, viscosity, diffusivity, density, and pressure are taken as constants when body forces are very small. In view of all the above conventions, the physical model in the Cartesian system will be expressed as

$$
\begin{aligned}
& \frac{\partial U}{\partial t^{*}}=v\left(1-K_{0} \frac{\partial}{\partial t^{*}}\right) \frac{\partial^{2} U}{\partial Y^{2}}+\frac{K_{1} N_{0}}{\rho}(V-U)-\frac{v}{K^{*}} U-\frac{\sigma_{e} B_{0}^{2}}{\rho} U+g \beta_{T}\left(T-T_{1}\right)+g \beta_{c}\left(C-C_{1}\right)+2 v \frac{\partial w^{*}}{\partial y}, \\
& m \frac{\partial V}{\partial t^{*}}=K_{1}(V-U), \\
& \frac{\partial T}{\partial t^{*}}=\frac{k}{\rho C_{P}} \frac{\partial^{2} T}{\partial Y^{2}}-\frac{Q_{T}}{\rho C_{P}}\left(T-T_{1}\right)-\frac{1}{\rho C_{P}} \frac{\partial q_{r}}{\partial Y}+\frac{v}{C_{P}}\left(\frac{\partial U}{\partial Y}\right)^{2}+\frac{\sigma_{e} B_{0}^{2}}{\rho C_{P}} U^{2}+\frac{Q_{c}}{\rho C_{P}}\left(C-C_{1}\right), \\
& \frac{\partial C}{\partial t^{*}}=D \frac{\partial^{2} C}{\partial Y^{2}}-K_{R}\left(C-C_{1}\right)+\frac{D K_{T}}{\bar{T}} \frac{\partial^{2} T}{\partial Y^{2}}, \\
& \frac{\partial C}{\partial t^{*}}=\frac{\alpha}{I} \frac{\partial^{2} w^{*}}{\partial Y^{2}} .
\end{aligned}
$$

The above governing equations are solved to find the flow field using the boundary conditions stated separately to both wavy as well as flat boundaries.

When $Y \in\left(\varepsilon^{*} \cos \left(K_{2} X\right), d\right)$ then, $t^{*}=0$ and $U=0, T=T_{1}$ and $V=0, c=c_{1}$

$Y=\varepsilon^{*} \cos \left(K_{2} X\right)$ then, $t^{*}>0$ and $U=0, C=C_{1}+\left(C_{2}-C_{1}\right) A t^{*}, V=0$,

also $-k \frac{\partial T}{\partial Y}=h_{f}\left(T_{1}-T+\left(T_{2}-T_{1}\right) A t^{*}\right)$.

when $Y=d$ then $U=U_{P}$ and $C=C_{2}+\left(C_{2}-C_{1}\right) A t^{*}, V=U_{P}$,

also $-k \frac{\partial T}{\partial Y}=h_{f}\left(T_{2}-T+\left(T_{2}-T_{1}\right) A t^{*}\right)$.

For the physical problem, the radiative heat flux is

$$
\frac{\partial q_{r}}{\partial Y}=4\left(T-T_{1}\right) I^{\prime}, I^{\prime}=\int_{0}^{\infty} K_{\lambda_{1} W} \frac{\partial e_{b \lambda_{1}}}{\partial T} d \lambda_{1} .
$$

The equations (1) to (5) are coupled nonlinear PDE's, for this, the non-dimensional parameters of the physical model are

$$
\begin{aligned}
& x=\frac{X}{d}, \quad y=\frac{Y}{d}, \quad v=\frac{V}{U_{0}}, \theta=\frac{T-T_{1}}{T_{2}-T_{1}}, \quad S_{c}=\frac{v}{d}, \quad A=\frac{U_{0}^{2}}{v}, \quad P_{r}=\frac{\mu C_{p}}{k}, \quad \phi=\frac{C-C_{1}}{C_{2}-C_{1}}, \\
& K_{r}=\frac{K_{R} d^{2}}{v}, E=\frac{K_{0} v}{d^{2}}, \alpha_{T}=\frac{Q_{T} d^{2}}{\mu C_{p}}, t=\frac{v t^{*}}{d^{2}}, S_{r}=\frac{D K_{T} d^{2}\left(T_{2}-T_{1}\right)}{v \bar{T}\left(C_{2}-C_{1}\right)}, M^{2}=\frac{\sigma_{e} B_{0}^{2} d^{2}}{\mu}, \frac{1}{K}=\frac{d^{2}}{K^{*}}, \\
& \delta=\frac{m N_{0}}{\rho}, F=\frac{4 I^{\prime} d^{2}}{\mu C_{p}}, W=\frac{m v}{K_{1} d^{2}}, G_{c}=\frac{g \beta_{c}\left(C_{2}-C_{1}\right) d^{2}}{v U_{0}}, B_{i}=\frac{h_{f} d}{k}, G_{r}=\frac{g \beta_{T}\left(T_{2}-T_{1}\right) d^{2}}{v U_{0}}, \\
& u_{p}=\frac{U_{p}}{U_{0}}, \varepsilon=\frac{\varepsilon^{*}}{d} \lambda=K_{2} d, h=\varepsilon \cos (\lambda x), \alpha_{c}=\frac{Q_{c} d^{2}}{\mu C_{p}} .
\end{aligned}
$$


On using the non-dimensional parameters (8) for the equations (1) to (5), the equations reduce to,

$$
\begin{aligned}
& \frac{\partial u}{\partial t}=\left(1-E \frac{\partial}{\partial t}\right) \frac{\partial^{2} u}{\partial y^{2}}+\frac{\delta}{w}(v-u)-\left(M^{2}+\frac{1}{k}\right) u+G_{r} \theta+G_{c} \phi-\Gamma u^{2}, \\
& w \frac{\partial v}{\partial t}=v-u, \\
& \frac{\partial \theta}{\partial t}=\frac{1}{p_{r}} \frac{\partial^{2} \theta}{\partial y^{2}}-\left(\alpha_{T}+F\right) \theta+E_{c} M^{2} u^{2}, \\
& \frac{\partial \phi}{\partial t}=\frac{1}{S_{c}} \frac{\partial^{2} \phi}{\partial y^{2}}-K_{r} \phi+S_{r} \frac{\partial^{2} \theta}{\partial y^{2}} .
\end{aligned}
$$

In view of equation (8) the dimensionless boundary conditions (6) to (7) reduces to the form when $y \in(h, 1)$ then $t=0$ and $\theta=0, \& u=0$ also $\phi=0$ and $V=0$,

when $y=h$ then $t>0$, and $\frac{\partial \theta}{\partial y}=B_{i}(\theta-t) \& u=0$ also $\phi=t$ and $v=0$,

when $y=1$ then $u=u_{p}$ and $\frac{\partial \theta}{\partial y}=B_{i}(\theta-1-t), \& v=u_{p}$, and $\phi=1+t$.

\section{Method of Solution}

Equations (9) to (13), are solved using perturbation method according to Prakash et al. [38], and the solution is assumed as

$$
\begin{aligned}
& u=u_{0}+S_{r} \Gamma e^{-n t} u_{1}+\text { order of }\left(\Gamma^{2}\right), \\
& v=v_{0}+S_{r} \Gamma e^{-n t} v_{1}+\text { order of }\left(\Gamma^{2}\right), \\
& \theta=\theta_{0}+S_{r} \Gamma e^{-n t} \theta_{1}+\text { order of }\left(\Gamma^{2}\right), \\
& \phi=\phi_{0}+S_{r} \Gamma e^{-n t} \phi_{1}+\text { order of }\left(\Gamma^{2}\right),
\end{aligned}
$$

On using the eqs. (14) to (17) for eqs. (9) to (13), we get below eqs.

$$
\begin{aligned}
& u_{0}^{\prime \prime}-N u_{0}=-\left(G_{r} \theta_{0}+G_{c} \phi_{0}\right), \\
& (1+n E) u_{1}^{\prime \prime}-\left(N-n+\frac{\delta n}{n+1}\right) u_{1}=-\left(G_{r} \theta_{1}+G_{c} \phi_{1}\right)+u_{0}^{2} e^{n t}, \\
& \theta_{0}^{\prime \prime}-P_{r}\left(\alpha_{T}+F\right) \theta_{0}=0, \\
& \theta_{1}^{\prime \prime}-P_{r}\left(\alpha_{T}+F-n\right) \theta_{1}=-P_{r}\left(\left(u_{0}^{\prime}\right)^{2}+M^{2} u_{0}^{2}\right), \\
& \phi_{0}^{\prime \prime}-K_{r} S_{c} \phi_{0}=-S_{r} S_{c} \theta_{0}^{\prime \prime}, \\
& \phi_{1}^{\prime \prime}-\left(K_{r}-n\right) S_{c} \phi_{1}=-S_{r} S_{c} \theta_{1}^{\prime \prime} .
\end{aligned}
$$

Also above conditions of the boundary take the form as when $y=h: u_{0}=0 ; \phi_{0}=t ; v_{0}=0 ; \theta_{0}^{\prime}=B_{i}\left(\theta_{0}-t\right)$,

also

$$
u_{1}=0 ; \phi_{1}=0 ; v_{1}=0 ; \theta_{1}^{\prime}=B_{i} \theta_{1} \text {. }
$$

when $y=1: u_{0}=u_{p} ; \phi_{0}=1+t ; v_{0}=u_{p} ; \theta_{0}^{\prime}=B_{i}\left(\theta_{0}-1-t\right)$, also

$$
u_{1}=0 ; \phi_{1}=0 ; v_{1}=0 ; \theta_{1}^{\prime}=B_{i} \theta_{1} \text {. }
$$


Further, the equations (18) to (24) are evaluated analytically to get the velocity of the fluid and dusty fluid also the temperature of the fluid, and the concentration of the fluid.

\section{Results and Discussion}

The system of equations is solved analytically using the perturbation method to obtain the expressions of the velocity of the dusty fluid, particle, temperature, and concentration for combined effects of radiation absorption and couple stress on Couette flow in a wavy channel for dusty viscoelastic fluid and the results are computed numerically and are shown graphically. These are some of the non-dimensional parameters which lead the model and are given as $\alpha_{T}$, $\mathrm{S}_{\mathrm{r}}, \mathrm{D}_{\mathrm{f}}, \Gamma, \mathrm{B}_{\mathrm{i}}, \mathrm{G}_{\mathrm{r}}, \mathrm{G}_{\mathrm{c}}, \mathrm{M}, \mathrm{P}_{\mathrm{r}}, \mathrm{K}_{\mathrm{r}}$. We have fixed few parameters throughout the model in order to maintain consistency as $\lambda=2 \pi, \mathrm{x}=1, \mathrm{G}_{\mathrm{r}}=10, \mathrm{G}_{\mathrm{c}}=10, \mathrm{P}_{\mathrm{r}}=5, \mathrm{~S}_{\mathrm{c}}=0.96, \mathrm{~K}_{\mathrm{r}}=1, \mathrm{~W}=3$. The variations of velocity in the case of dusty fluid and particle, temperature, and concentration of several factors are displayed graphically in Figures 2 to 27.

Figureures 2 to 4 address the influence of heat absorption factors for a couple of stress fluids through a wavy channel. It is observed from Figure 2 that as the heat absorption factor increases, there is a fall in the velocity of the dusty fluid and particle further in case of temperature as the heat absorption factor enhances, there is a rise in the temperature initially. It reduces as it progresses towards the channel due to the channel resistance and is depicted in Figure 3. There is a fall in the concentration profile due to the rise in the heat absorption factor, as shown in Figure 4. The velocity of the dusty fluid and the particle, temperature, and concentration of the Soret parameter are shown in Figures 5 to 7. As the Soret number enhances, velocity, temperature, and concentration of the fluid also increase because of higher temperature difference, but concentration variance is fixed, or it can be because of reduction in concentration difference, while temperature variance is fixed. The effect of Biot number on velocity, temperature, and concentration can be depicted in Figures 8 to 10. From Figure 8, it is clear that the velocity of dusty fluid and particle reduces as there is a rise in Biot number, and this can be due to the greater thermal conductivity. The effects of temperature and concentration on Biot number is shown in Figure 9 and 10.

For the higher solutal Grashoff number, shrink in the velocity of dusty fluid and particle is depicted in Figure 11 due to buoyancy. From Figure 12, it is clear that there is a fall in the fluid temperature, as the solutal Grashoff parameter is raised because of variation in temperature of the wall and thermal expansion. Figure 13 shows that there is a decrease in the concentration of the fluid as the solutal Grashof parameter is increased. From Figure 14, it is well-known that the magnetic field enhances the velocity of the dusty fluid, and particle diminishes because of the effect of resistive force called Lorentz force. The effect of the velocity of fluid and particle when the Prandtl number is elevated is observed in Figure 15, and due to this, there is a fall in the velocity. In Figure 16, it can be witnessed that as the Prandtl quantity enlarges, the temperature lowers because of thermal conductivity and a denser boundary layer. The concentration decreases as the Prandtl number is raised shown in Figure 17. Due to the rise in the chemical reaction, the velocity and temperature of the fluid falls and are witnessed in Figures 18 and 19.

In concentration outlines, it is well-known that as the chemical reaction factor improves, a drop in the fluid's concentration is viewed. This is because of the variation of diffusion, which controls the chemical reaction constraint. This can be witnessed in Figure 20. As the porous permeability factor is improved, the velocity of the dusty fluid and the particle 
reduces because of porosity, and the improvement of its viscosity is revealed in Figure 21. In Figures 22 and 23, we can observe that as porous permeability raises, the fluid's temperature and concentration reduce. From Figure 24, it is observed that as the couple stress factor raises, the velocity increases. As the radiation absorption parameter enhances, there is an increase in the velocity of the dusty fluid and particle, as in Figure 25. The temperature of the fluid rises as the radiation absorption quantity raises shown in Figure 26. The fluid concentration enhances as the radiation absorption parameter rises due to the solutal difference and absorption coefficient, shown in Figure 27.

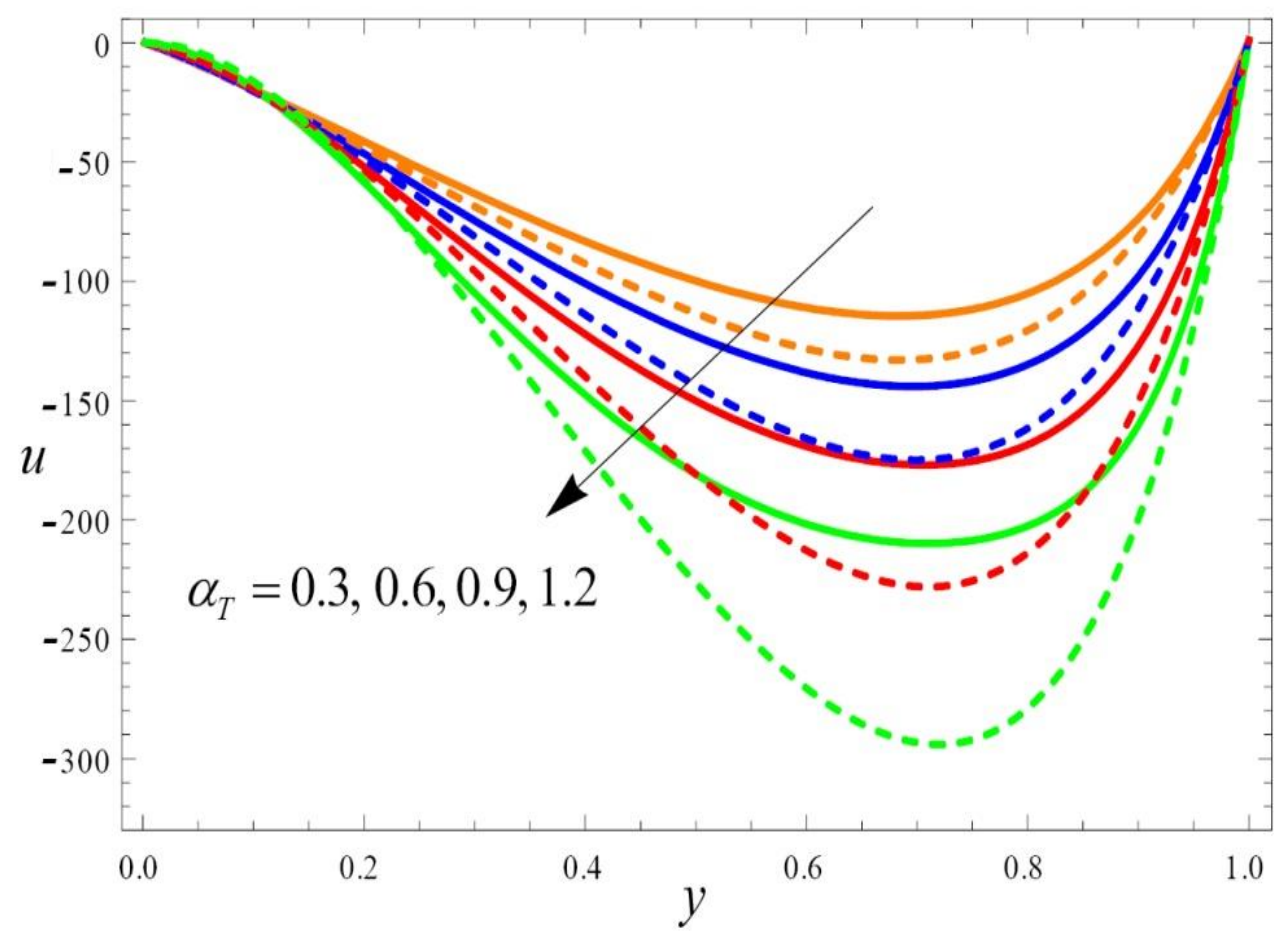

Figure 2. Velocity profiles of heat absorption parameter.

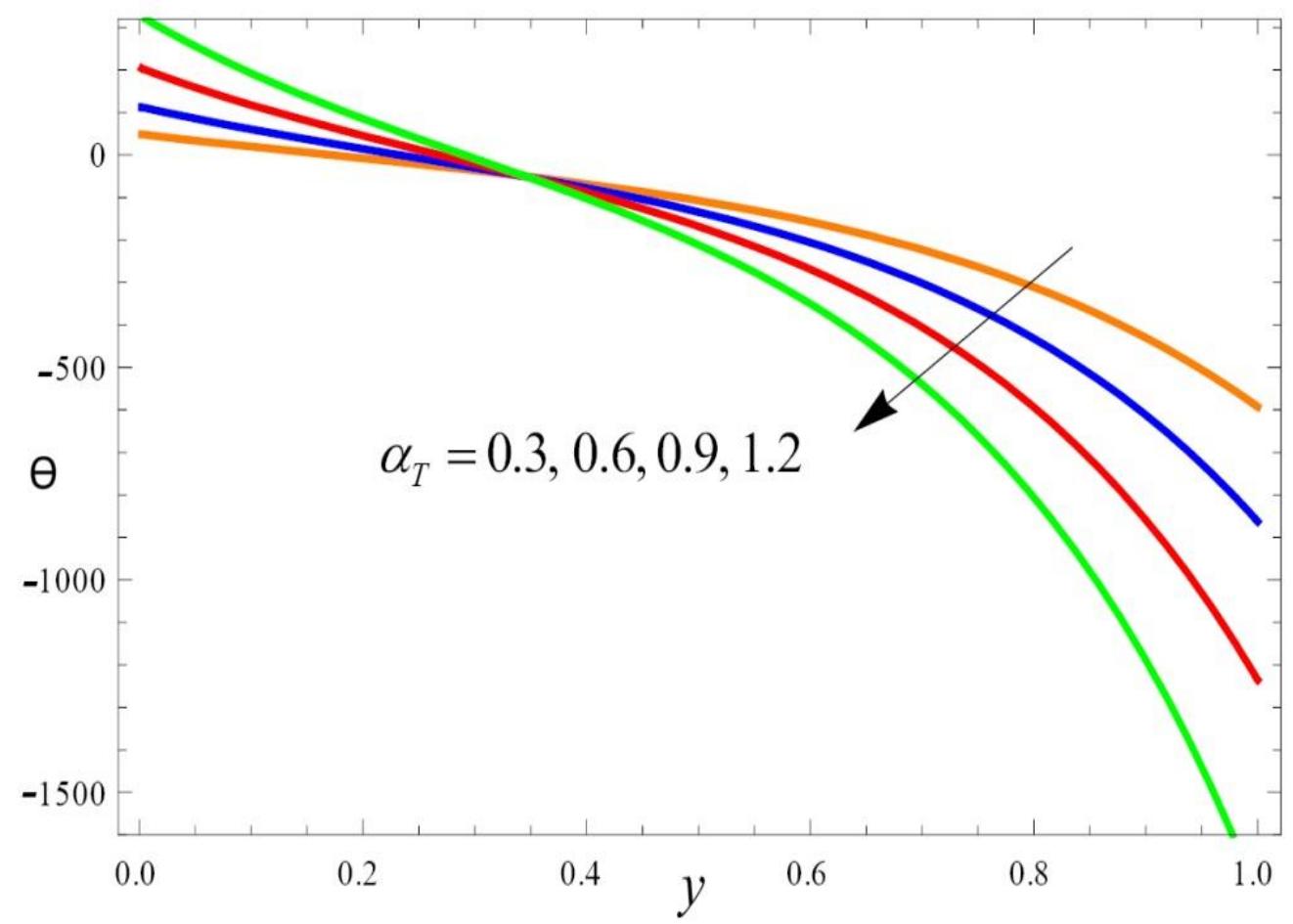

Figure 3. Temperature profiles of heat absorption parameter. 


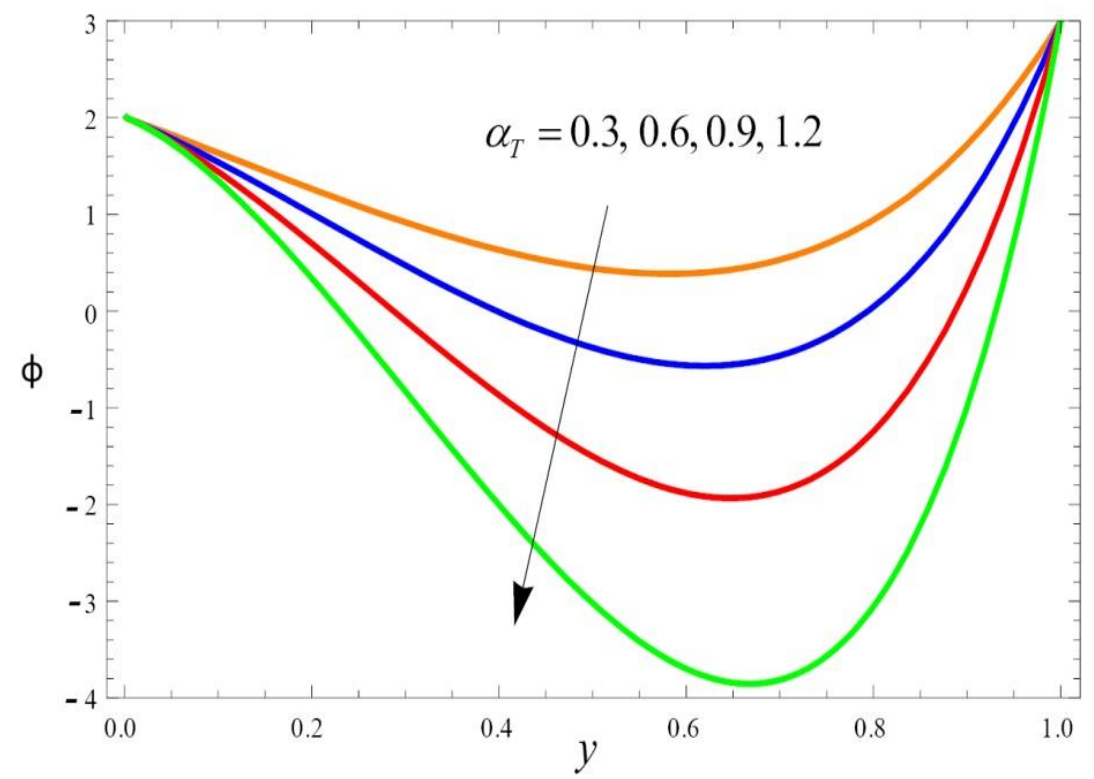

Figure 4. Concentration profiles of heat absorption parameter.

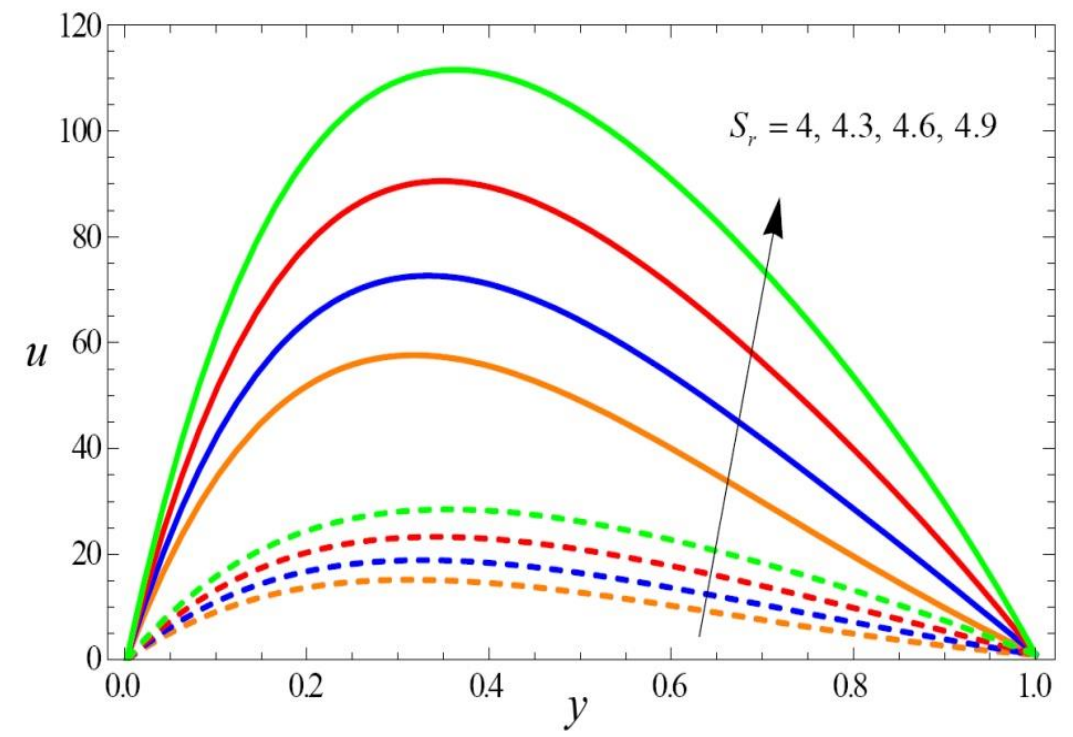

Figure 5. Velocity profiles of Soret parameter.

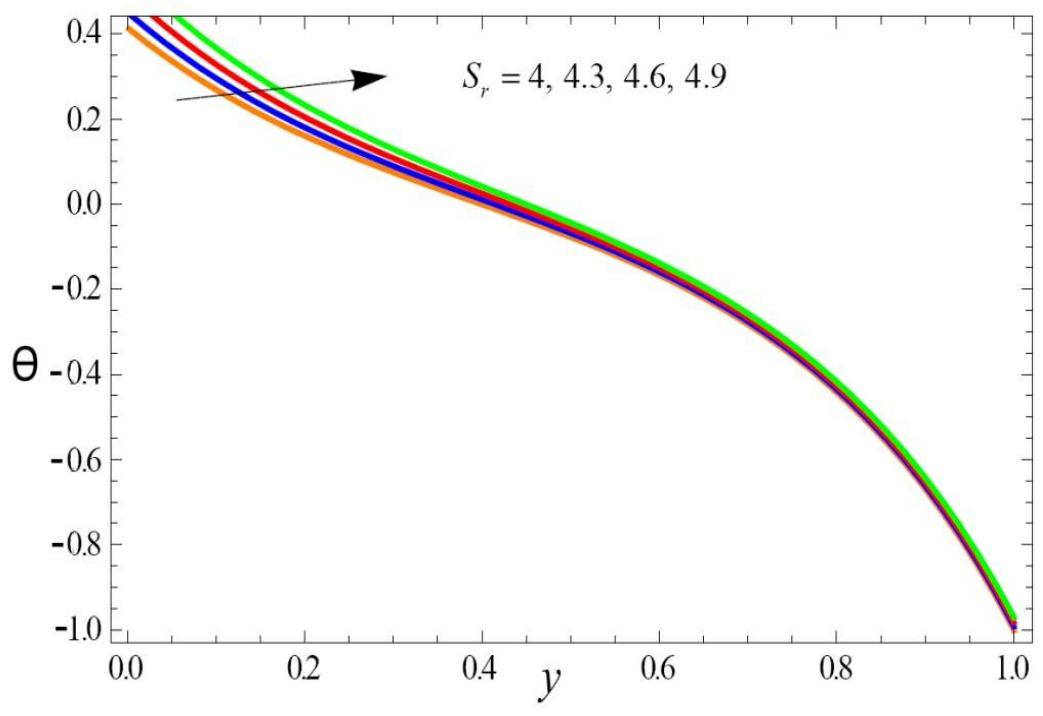

Figure 6. Temperature profiles of Soret parameter. 


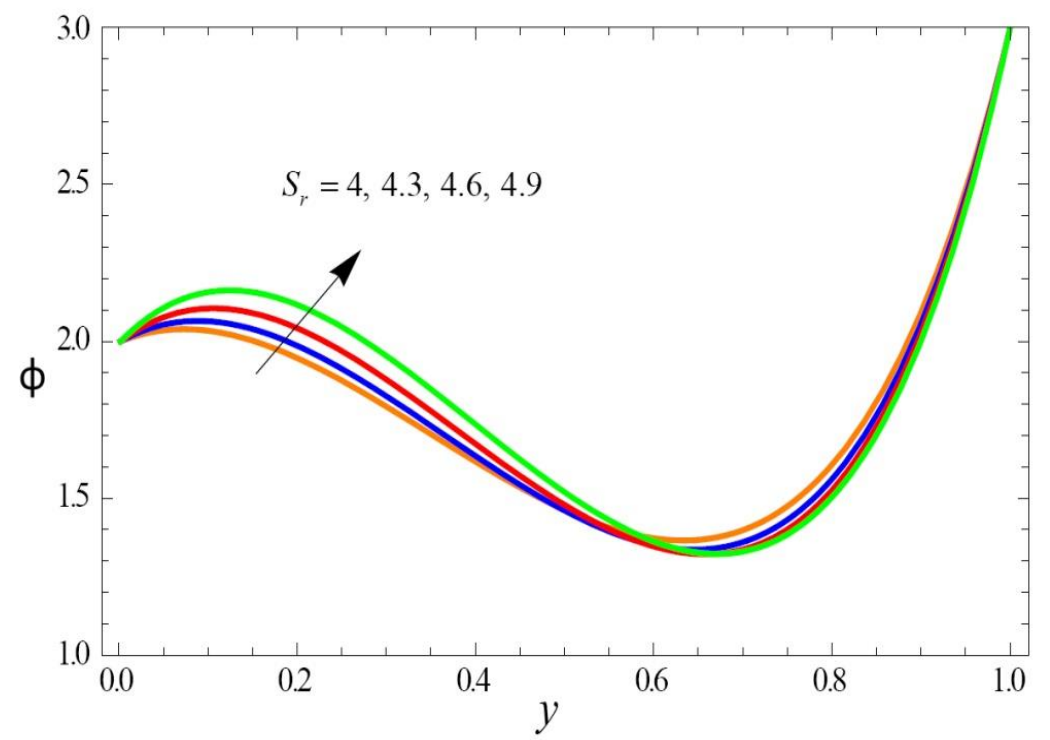

Figure 7. Concentration profiles of Soret parameter.

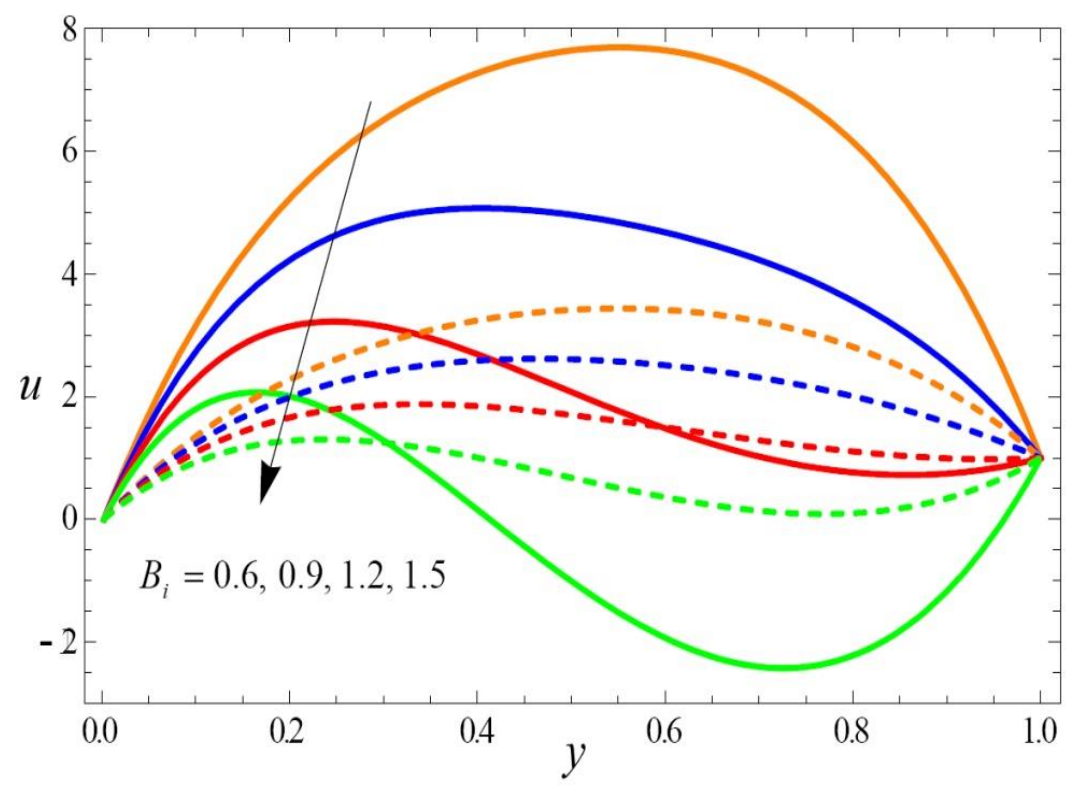

Figure 8. Velocity profiles of Biot number.

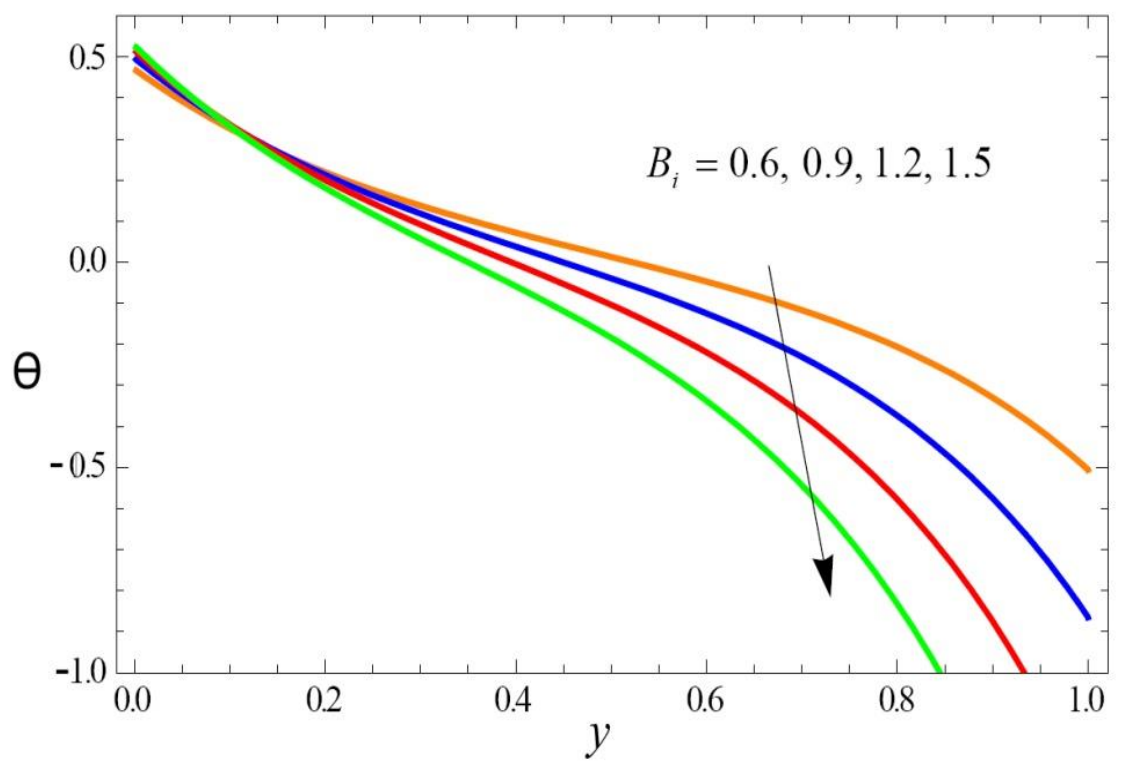

Figure 9. Temperature profiles of Biot parameter. 


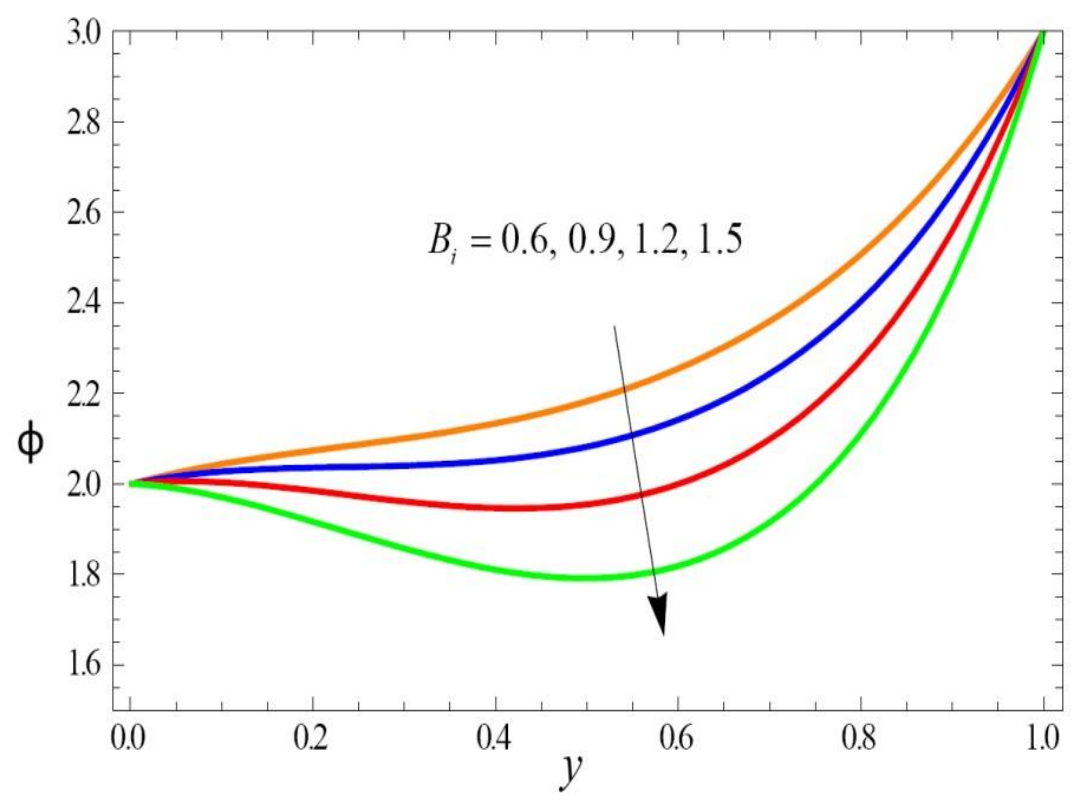

Figure 10. Concentration profiles of Biot number.

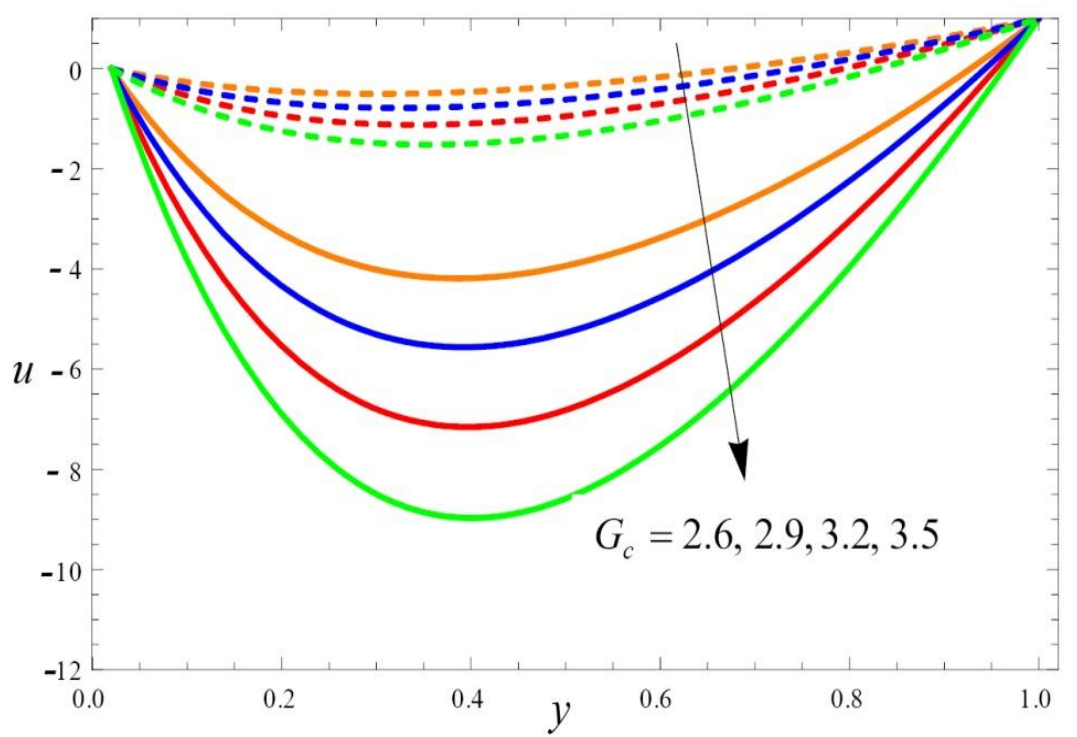

Figure 11. Velocity profiles of solutal Grashof number.

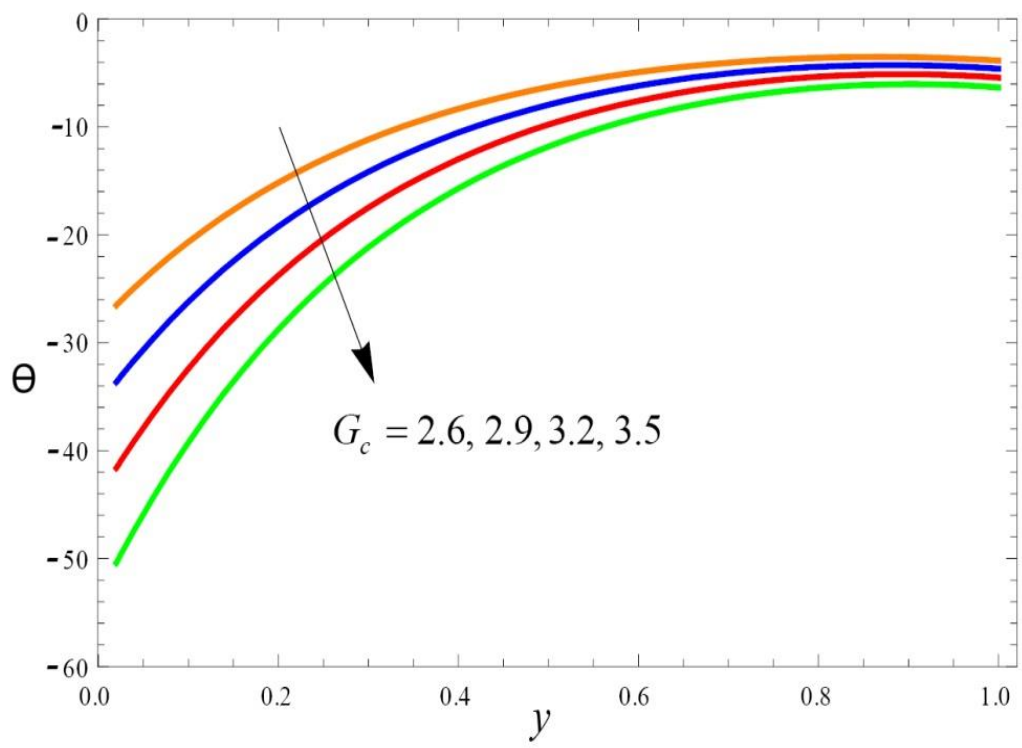

Figure 12. Temperature profiles of solutal Grashof number. 


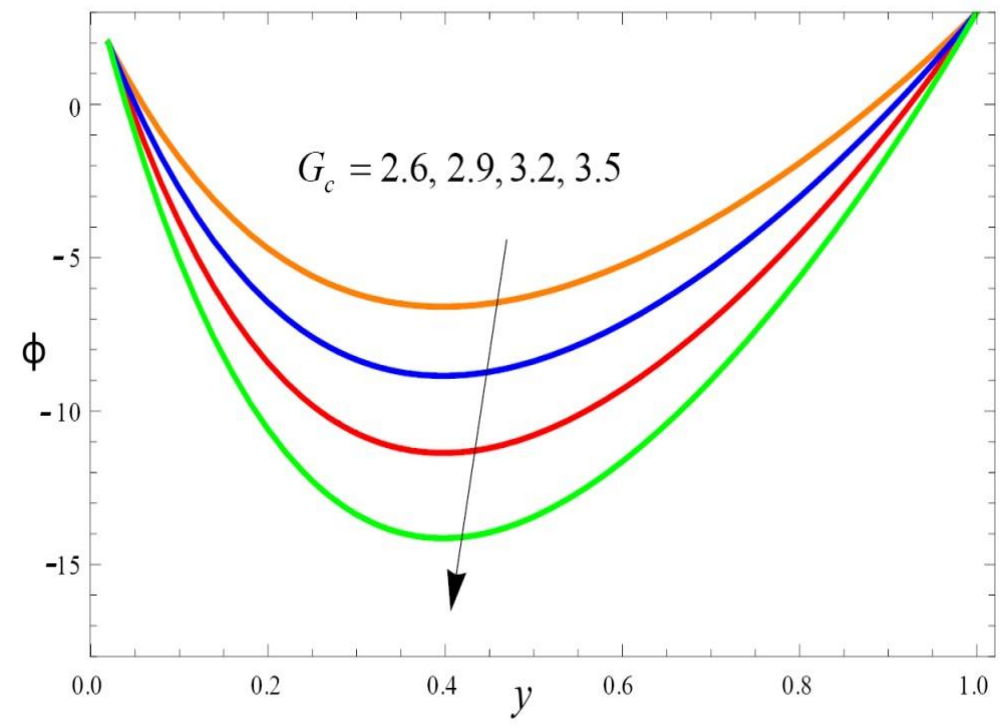

Figure 13. Concentration profiles of solutal Grashof number.

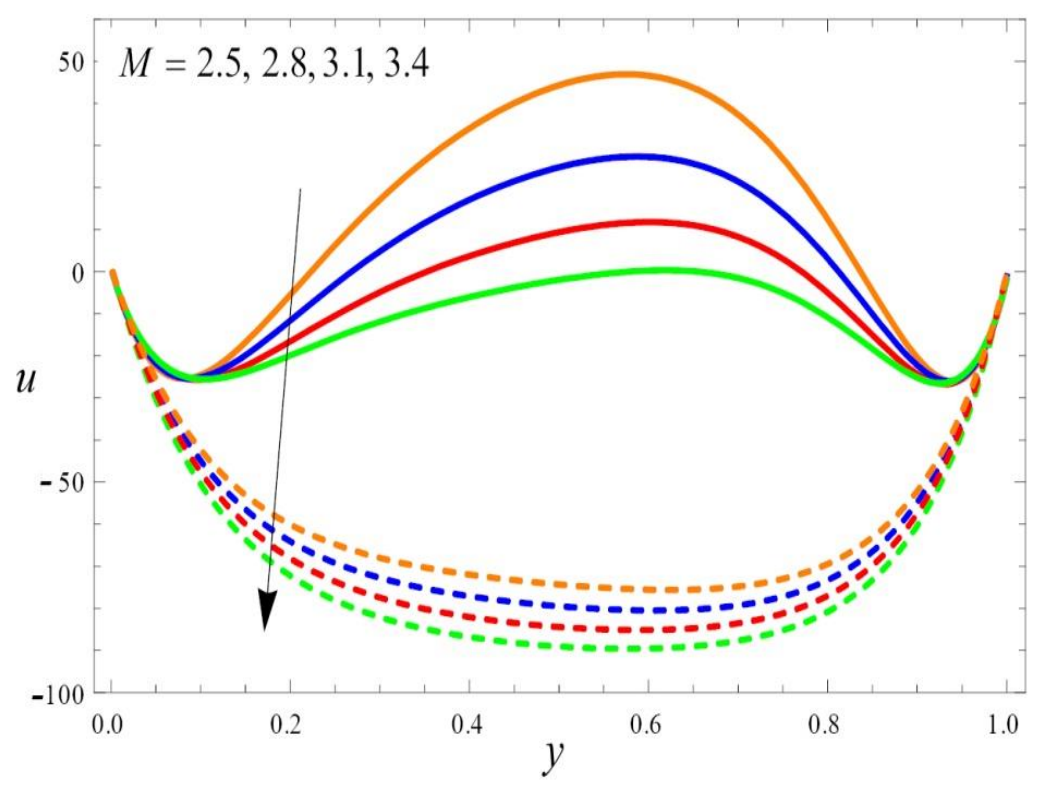

Figure 14. Velocity profiles of Magnetic parameter.

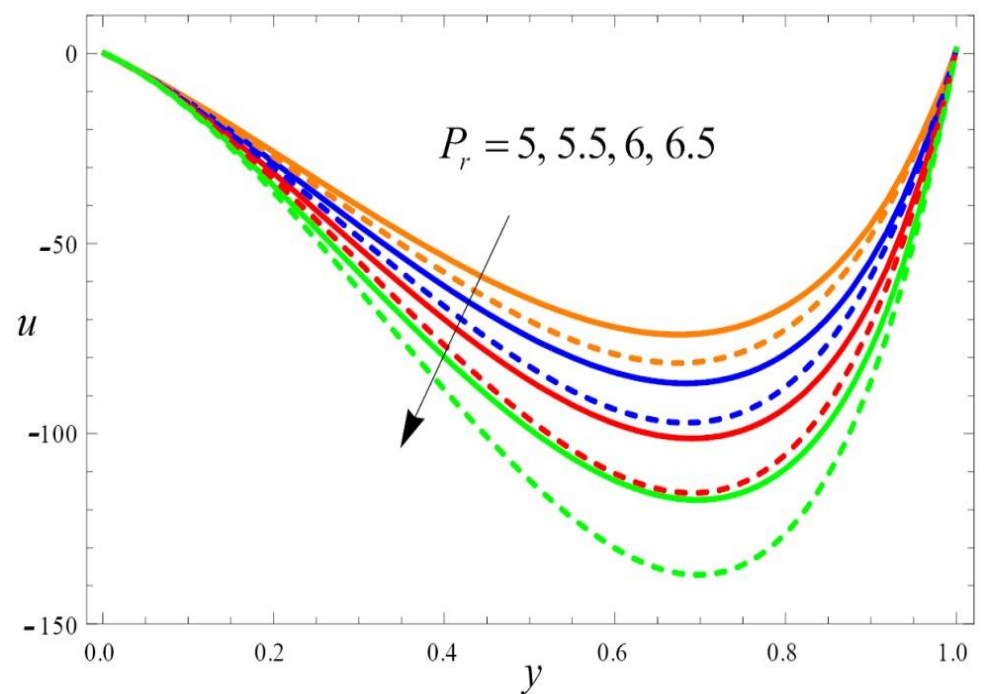

Figure 15. Velocity profiles of Prandtl number. 


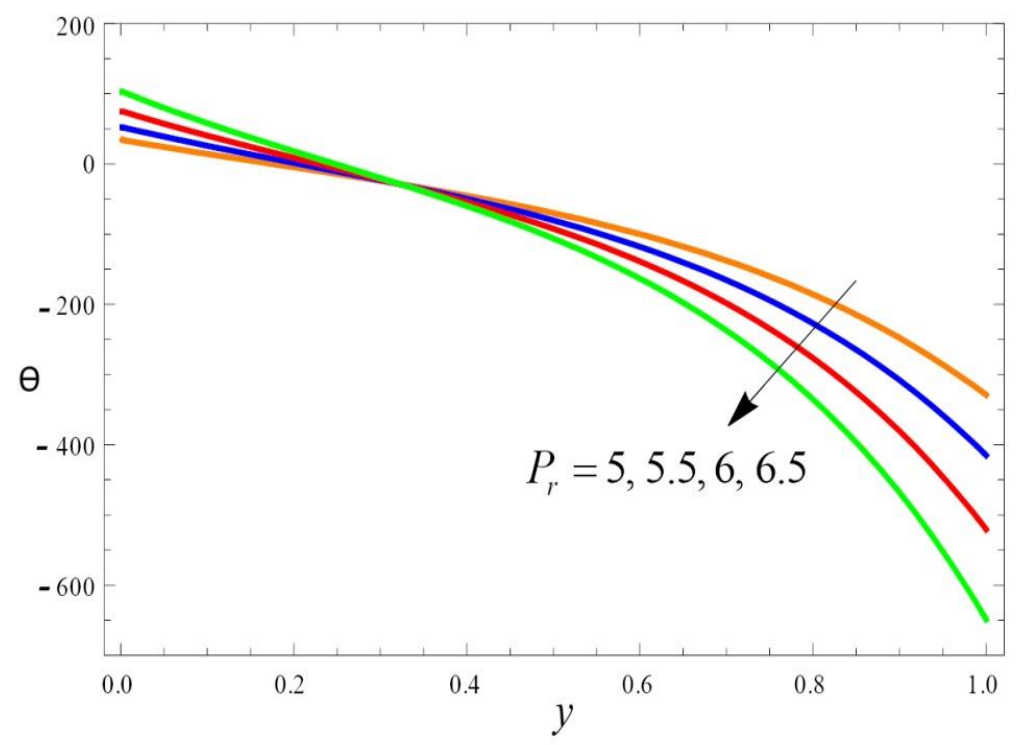

Figure 16. Temperature profiles of Prandtl number.

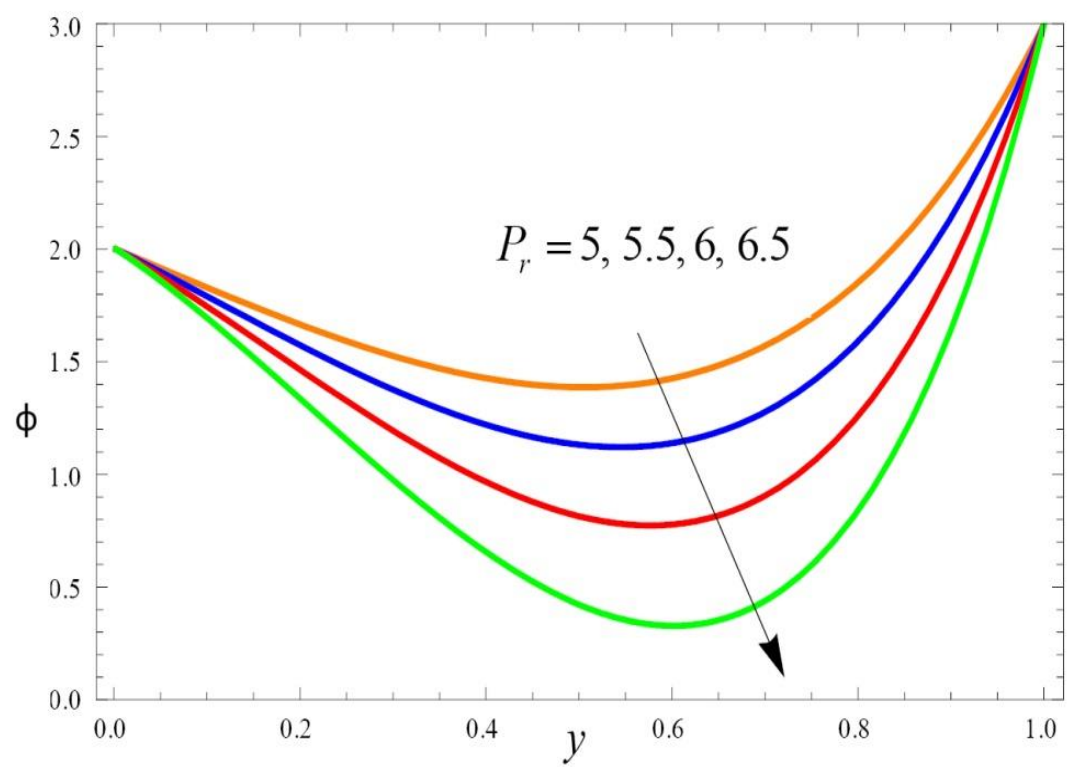

Figure 17. Concentration profiles of Prandtl number.

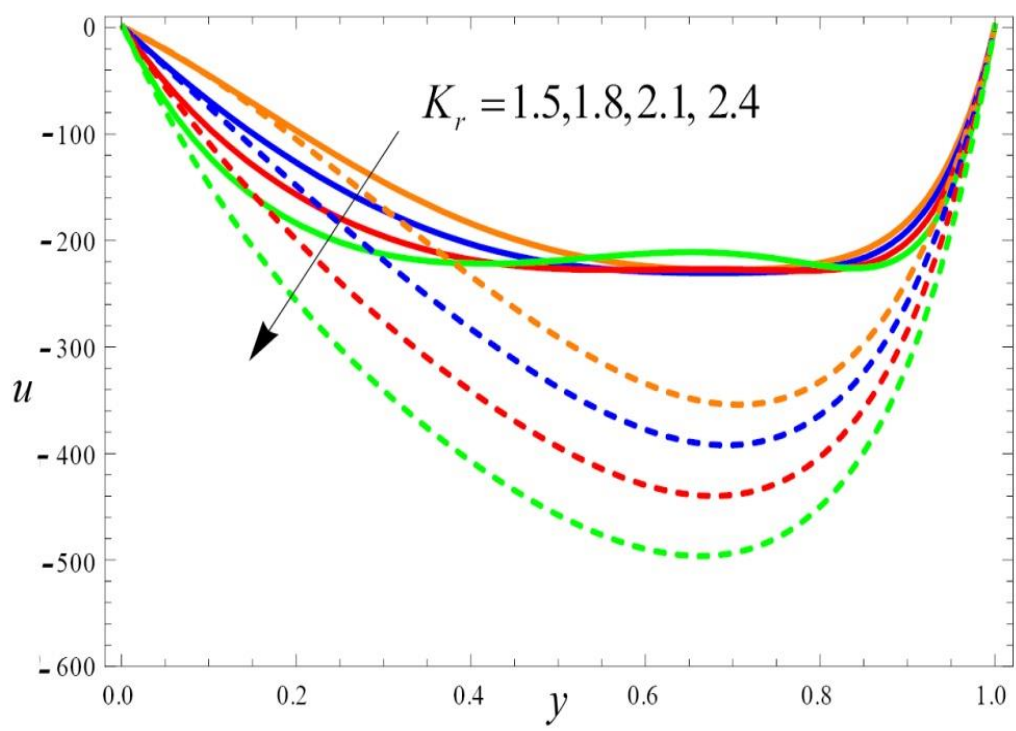

Figure 18. Velocity profiles of chemical reaction parameter. 


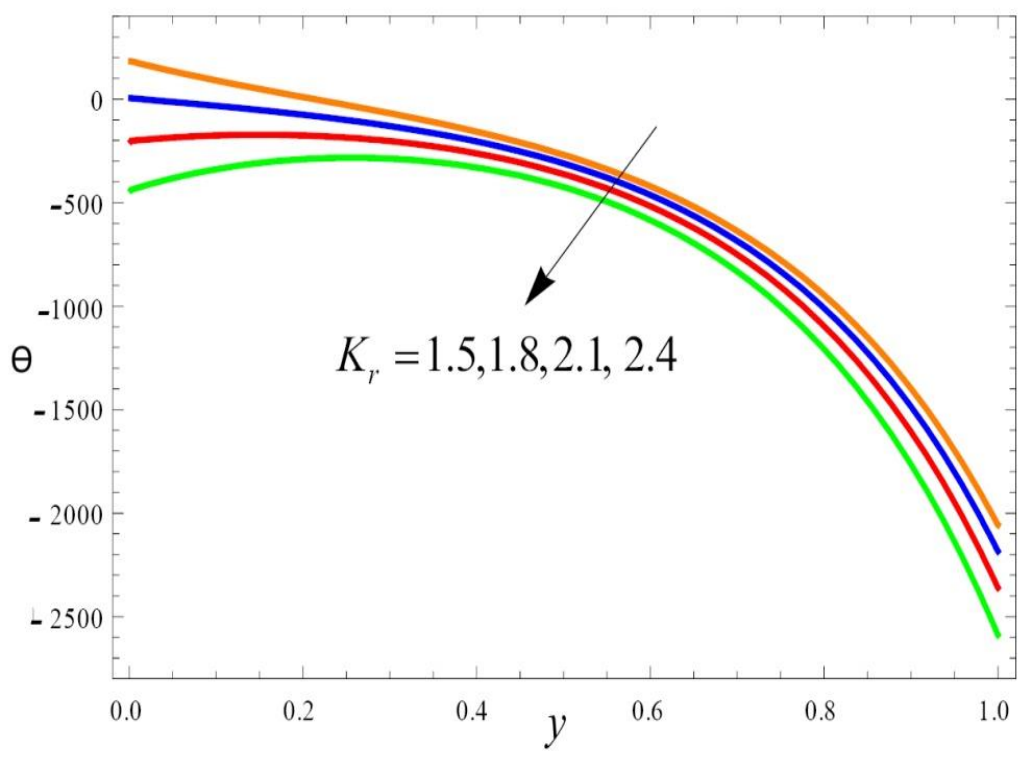

Figure 19. Temperature profiles of chemical reaction parameters.

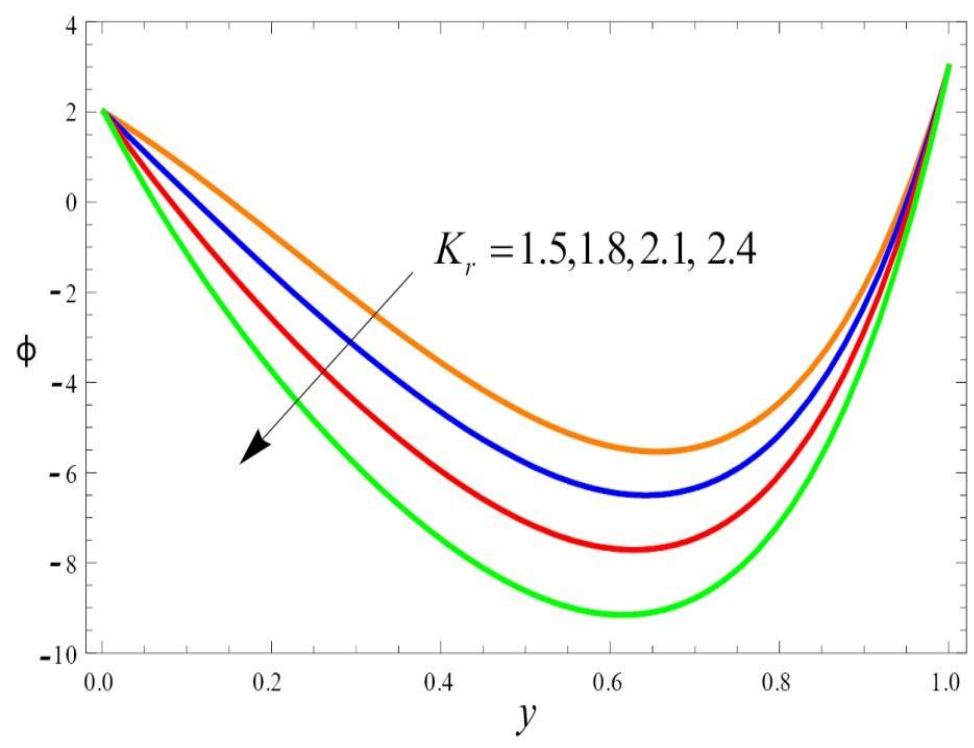

Figure 20. Concentration profiles of chemical reaction parameters.

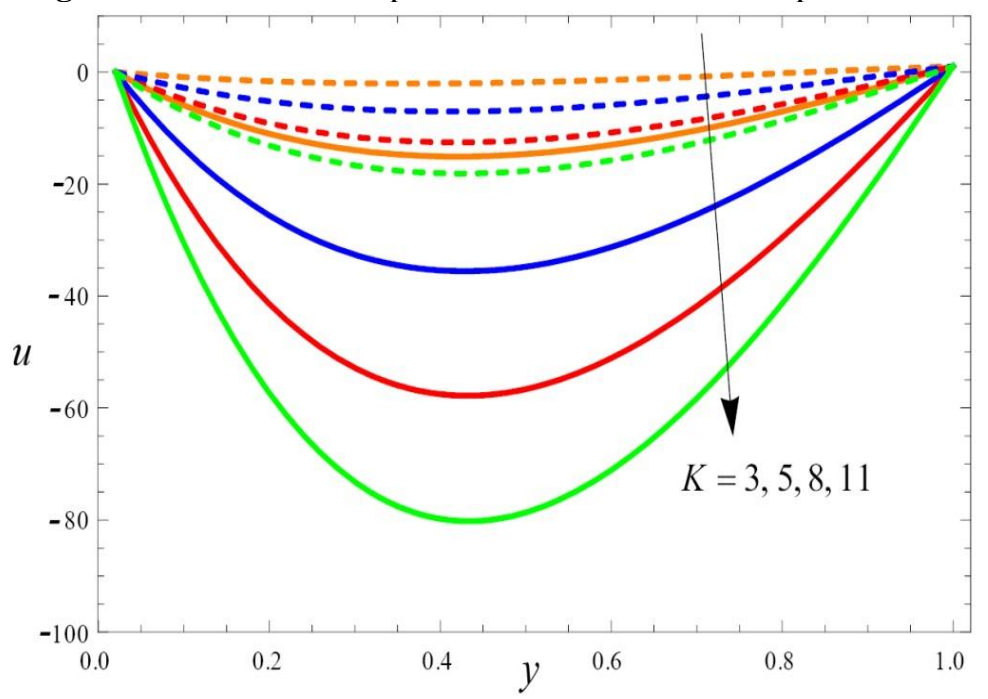

Figure 21. Velocity profiles of porous permeability parameter. 


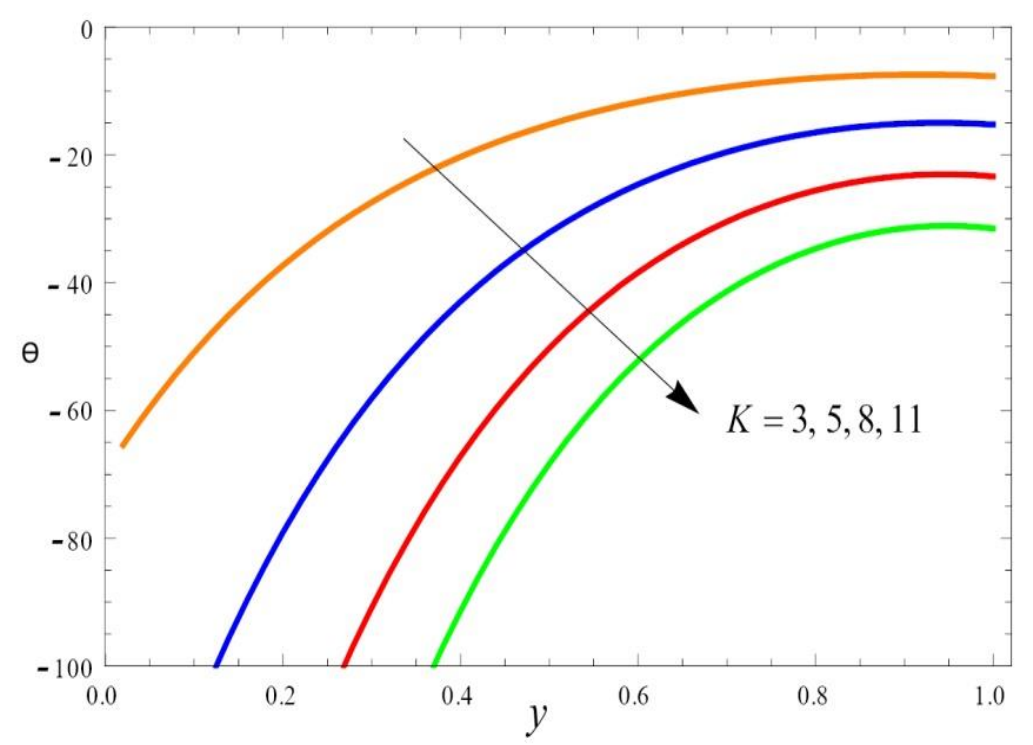

Figure 22. Temperature profiles of porous permeability parameter.

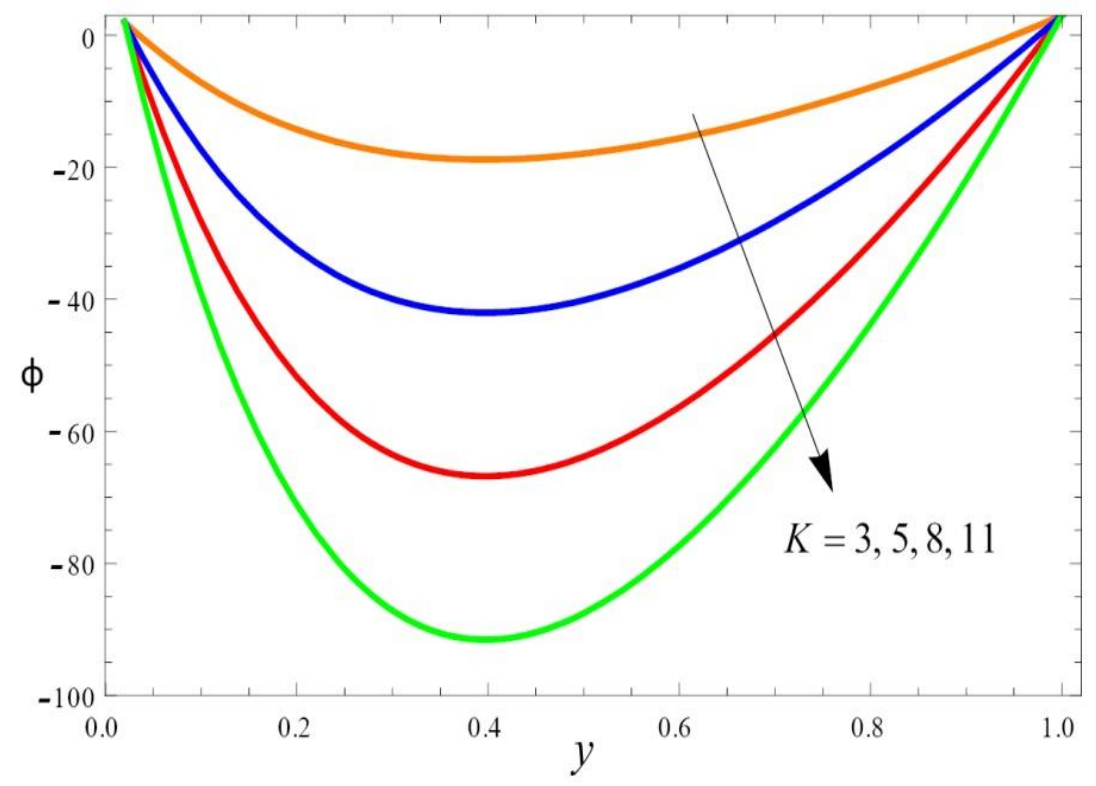

Figure 23. Concentration profiles of porous permeability parameter.

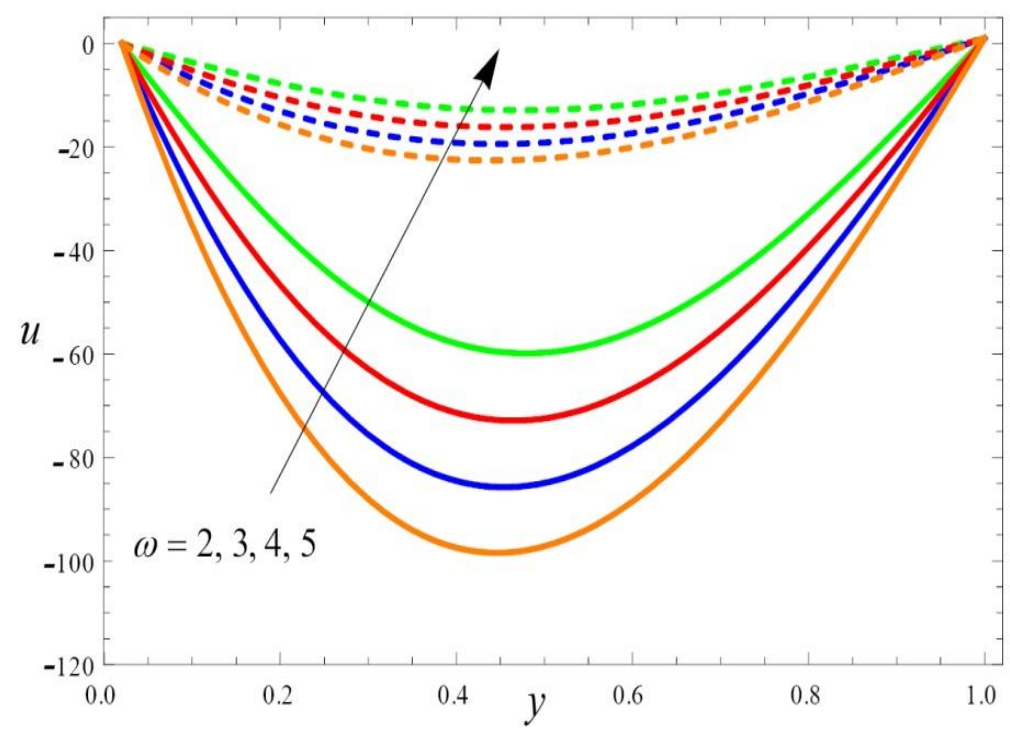

Figure 24. Velocity profiles of couple stress parameters. 


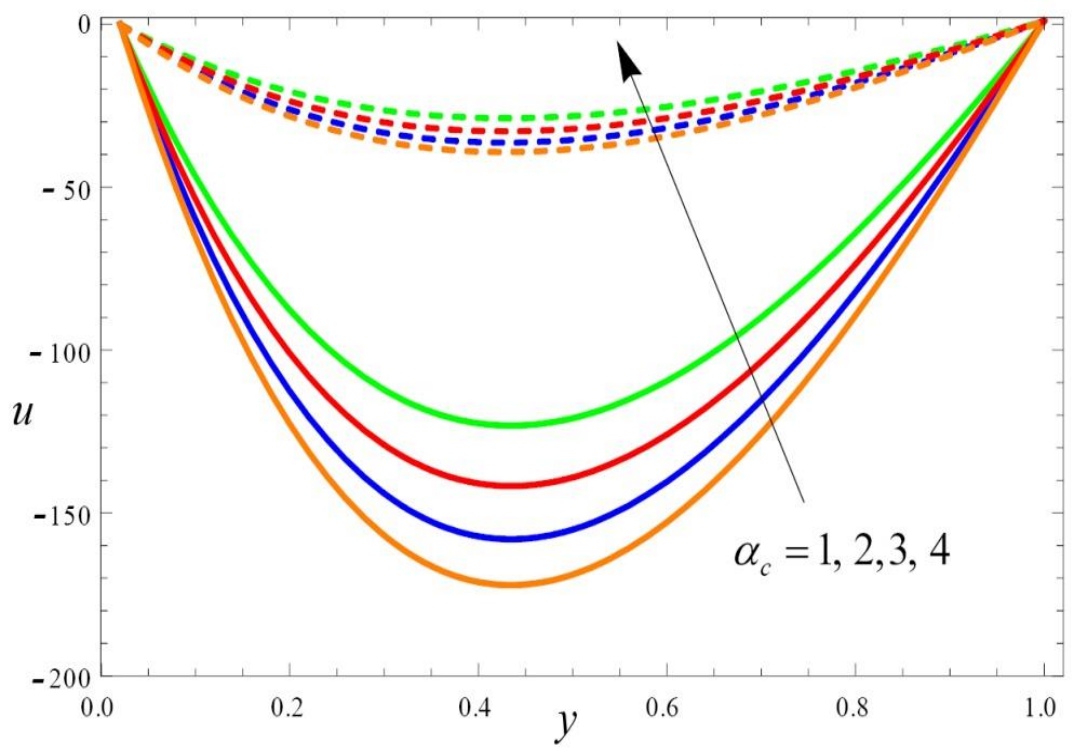

Figure 25. Velocity profiles of radiation absorption parameter.

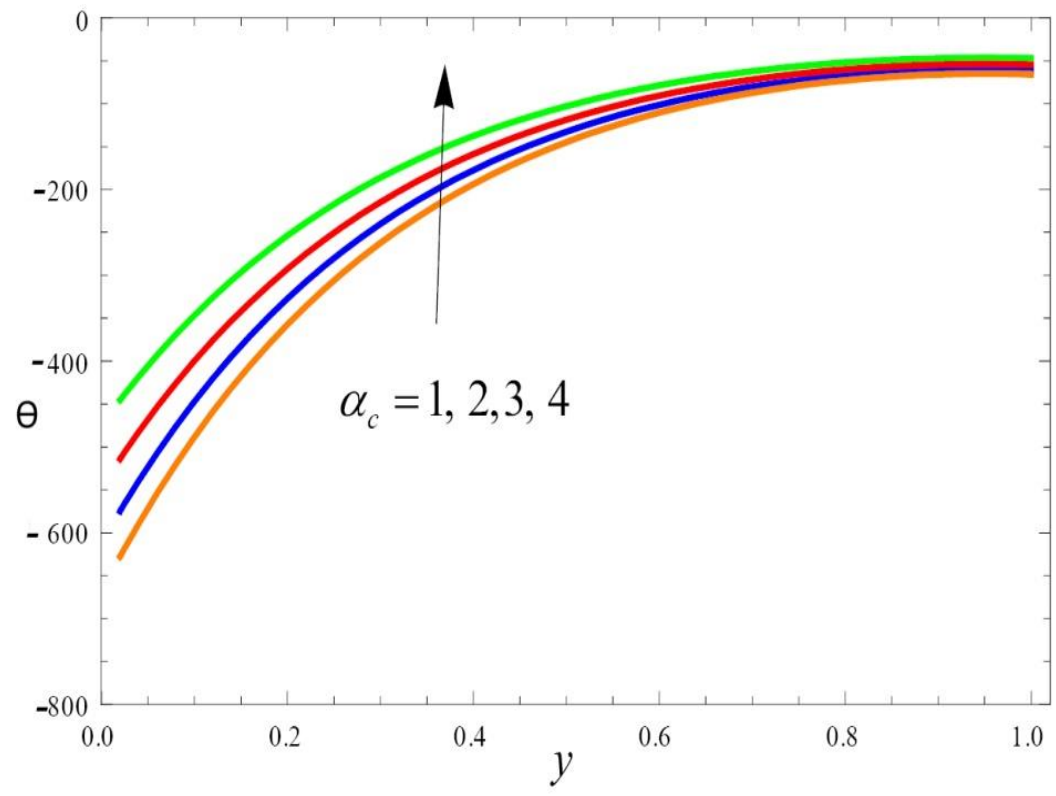

Figure 26. Temperature profiles of radiation absorption parameter.

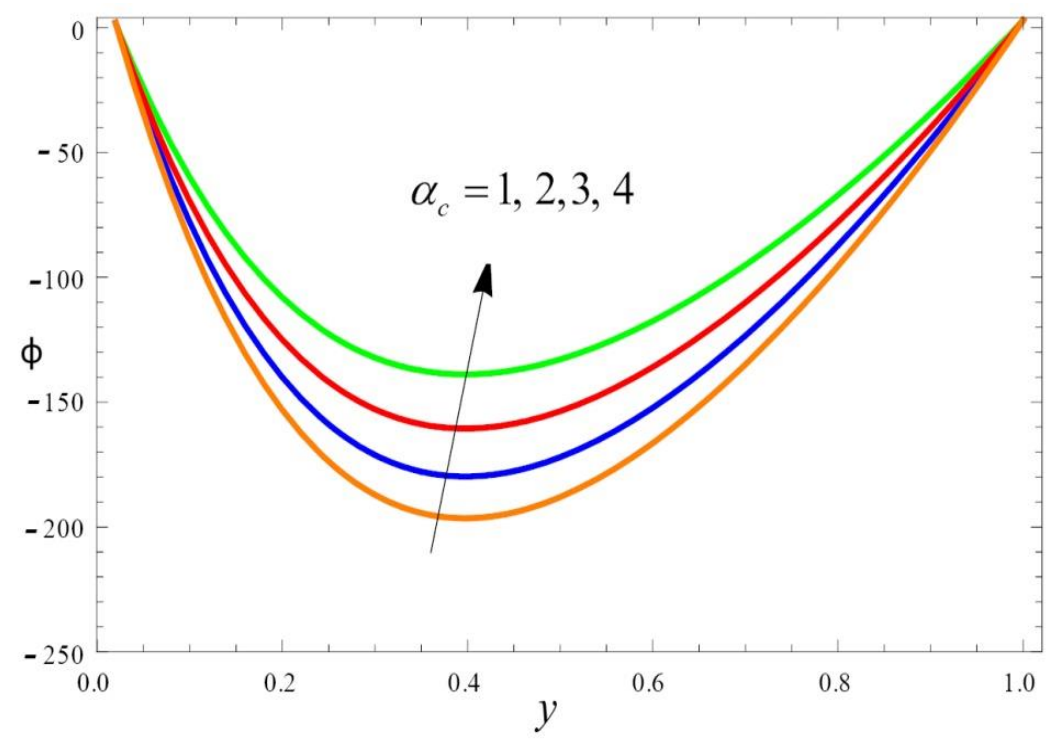

Figure 27. Concentration profiles of radiation absorption parameter. 


\section{Conclusions}

In the current work, an effort is made to examine the effects of couple stress, Soret, radiation absorption parameter, heat absorption parameter, etc., on Couette flow in a wavy channel for dusty fluid. To resolve the physical model, an analytical perturbation method is used. The results are computed numerically and are displayed graphically. The current research study allows us to draw the following conclusions: (i) As the couple stress of the fluid raises, there is a rise in the velocity of the fluid; (ii) As the magnetic field enhances, the velocity of both dusty fluid and particle shrinks; (iii) With raise of radiation absorption, the velocity, temperature, and concentration profiles increases; (iv) There is an enhancement in velocity, temperature and concentration of fluid as Soret number increases; (v) As the chemical reaction factor is increased there is a decrease in velocity of the fluid, particle, temperature, and concentration of viscoelastic fluid; (vi) The profiles of velocity reduce near the wavy wall; (vii) As the heat absorption parameter enhances there will be a decrease in velocity of fluid, particle, temperature, and concentration of the fluid.

\section{Funding}

This research received no external funding.

\section{Acknowledgments}

The authors would like to thank the anonymous reviewers for their valuable comments and suggestions to improve the quality of the paper.

\section{Conflicts of Interest}

The authors declare no conflict of interest.

\section{References}

1. Walters, K. Non-Newtonian effects in some elastico-viscous liquids whose behaviour at small rates of shear is characterized by a general linear equation of state. The Quarterly Journal of Mechanics and Applied Mathematics 1962, 15, 63-76, https://doi.org/10.1093/qjmam/15.1.63.

2. Attia, H.A. Unsteady MHD Couette flow and heat transfer of dusty fluid with variable physical properties. Applied Mathematics and computation 2006, 177, 308-318, https://doi.org/10.1016/j.amc.2005.11.010.

3. Mekheimer, K.S. The influence of heat transfer and magnetic field on peristaltic transport of a Newtonian fluid in a vertical annulus: application of an endoscope. Physics letters A 2008, 372, 1657-1665, https://doi.org/10.1016/j.physleta.2007.10.028.

4. Ashmawy, E.A. Unsteady Couette flow of a micropolar fluid with slip. Meccanica 2012, 47, 85-94, https://doi.org/10.1007/s11012-010-9416-7.

5. Wang, Z.J.; Jourdan, E. Benchmark for scale-resolving simulation with curved walls: the Taylor Couette flow. Advances in Aerodynamics 2021, 3, 1-18, https://doi.org/10.1186/s42774-021-00071-0.

6. Uma, M.; Dinesh, P.A. Effects of radiation absorption, Soret and Dufour on couette flow in an irregular channel for dusty viscoelastic fluid. International Journal of Science, Technology, Engineering and Management-A 2019, 1, 24-30.

7. Dinesh, P.A.; Vasudevamurthy, A.S.; Uma, M. Effects of Forchheimer, MHD and Radiation Absorption for Chemically Reacting Unsteady Dusty Viscoelastic Fluid Couette Flow in an Irregular Channel. In Advances in Fluid Dynamics, Springer, Singapore 2021, 999-1012, https://doi.org/10.1007/978-981-15-4308.

8. Deb, H. R. Hydromagnetic oscillatory couette flow of a visco-elastic fluid with dust in a channel with radiative heat. In Emerging Technologies in Data Mining and Information Security, Springer, Singapore 2021, 105-115, https://doi.org/10.1007/978-981-15-9927-9_11.

9. Sharma, T.; Sharma, P.; Kumar, N. Entropy generation in thermal radiative oscillatory MHD couette flow in the influence of heat source. In Journal of Physics: Conference Series 2021, 012023, https://doi.org/10.1088/1742-6596/1849/1/012023. 
10. Gireesha, B.J.; Chamkha, A.J.; Vishalakshi, C. S.; Bagewadi, C. S. Three-dimensional Couette flow of a dusty fluid with heat transfer. Applied Mathematical Modelling 2012, 36, 683-701, https://doi.org/10.1016/j.apm.2011.07.014.

11. Harish Babu, D.; Satya Narayana, P.V. Influence of variable permeability and radiation absorption on heat and mass transfer in MHD micropolar flow over a vertical moving porous plate. International Scholarly Research Notices 2013, http://dx.doi.org/10.1155/2013/953536.

12. Devika, B.; Narayana, P. S.; Venkataramana, S. Chemical reaction effects on MHD free convection flow in an irregular channel with porous medium. International Journal of Mathematical Archive 2013, 4, 282-295, http://www.ijma.info/index.php/ijma/article/view/2065.

13. Sheikholeslami, M.; Hatami, M.; Ganji, D. D. Micropolar fluid flow and heat transfer in a permeable channel using analytical method. Journal of Molecular Liquids 2014, 194, 30-36, https://doi.org/10.1016/j.molliq.2014.01.005.

14. Ahmed, S.; Zueco, J.; López-González, L. M. Numerical and analytical solutions for magnetohydrodynamic 3D flow through two parallel porous plates. International Journal of Heat and Mass Transfer 2017, 108, 322331, https://doi.org/10.1016/j.ijheatmasstransfer.2016.11.102.

15. Venkateswarlu, B.; Narayana, P.S.; Devika, B. Effects of chemical reaction and heat source on MHD oscillatory flow of a viscoelastic fluid in a vertical porous channel. International Journal of Applied and Computational Mathematics 2017, 3, 937-952, https://doi.org/10.1007/s40819-017-0391-8.

16. Job, V.M.; Gunakala, S.R. Finite element analysis of unsteady radiative MHD natural convection Couette flow between permeable plates with viscous and joule dissipation. International Journal of Pure and Applied Mathematics 2015, 99, 123-143, https://dx.doi.org/10.12732/ijdea.v12i1.823.

17. Uma, M.; Dinesh, P.A. Combined effects of Forchheimer, Soret and Dufour on MHD mixed convective dusty viscoelastic Couette flow in an irregular channel. Multidiscipline Modeling in Materials and Structures 2020, 17, 49-64, https://doi.org/10.1108/MMMS-12-2019-0233.

18. Shankar, B.M.; Shivakumara, I.S.; Kumar, J. Benchmark solution for the hydrodynamic stability of plane porous-Couette flow. Physics of Fluids 2020, 32, 104104, https://doi.org/10.1063/5.0014093.

19. Aydinbakar, L.; Takizawa, K.; Tezduyar, T.E.; Kuraishi, T. Space-time VMS isogeometric analysis of the Taylor-Couette flow. Computational Mechanics 2021, 67, 1515-1541, https://doi.org/10.1007/s00466-02102004-6.

20. Reddy, M.G.; Reddy, K.V.; Makinde, O. D. Hydromagnetic peristaltic motion of a reacting and radiating couple stress fluid in an inclined asymmetric channel filled with a porous medium. Alexandria Engineering Journal 2016, 55, 1841-1853 https://doi.org/10.1016/j.aej.2016.04.010.

21. Hayat, T.; Muhammad, T.; Qayyum, A.; Alsaedi, A.; Mustafa, M. On squeezing flow of nanofluid in the presence of magnetic field effects. Journal of Molecular Liquids 2016, 213, 179-185, https://doi.org/10.1016/j.molliq.2015.11.003.

22. Suresh Babu, R.; Kumar, B.R.; Dinesh, P.A. Effects of Mixed Convection on the Oscillatory Flow of a Couple Stress Fluid through a Vertical Plate with Variable Fluid Properties. Defect and Diffusion Forum 2018, 388, 328-343, https://doi.org/10.4028/www.scientific.net/DDF.388.328.

23. Malashetty, M.S.; Gaikwad, S.N.; \& Swamy, M. An analytical study of linear and nonlinear double diffusive convection with Soret effect in couple stress liquids. International journal of thermal sciences 2006, 45, 897907, https://doi.org/10.1016/j.ijthermalsci.2005.12.005.

24. Sohail, M.; Ali, U.; Al-Mdallal, Q.; Thounthong, P.; Sherif, E.S.M.; Alrabaiah, H.; Abdelmalek, Z. Theoretical and numerical investigation of entropy for the variable thermophysical characteristics of couple stress material: Applications to optimization. Alexandria Engineering Journal 2020, 59, 4365-4375, https://doi.org/10.1016/j.aej.2020.07.042.

25. Ali, R., Farooq, A.; Shahzad, A.; Benim, A.C.; Iqbal, A.; Razzaq, M. Computational approach on threedimensional flow of couple-stress fluid with convective boundary conditions. Physica A: Statistical Mechanics and its Applications 2020, 553, 124056, https://doi.org/10.1016/j.physa.2019.124056.

26. Fan, F.; Xu, Y.; Sahmani, S.; Safaei, B. Modified couple stress-based geometrically nonlinear oscillations of porous functionally graded microplates using NURBS-based isogeometric approach. Computer Methods in Applied Mechanics and Engineering 2020, 372, 113400, https://doi.org/10.1016/j.cma.2020.113400.

27. Liu, H.; Shen, S.; Oslub, K.; Habibi, M.; Safarpour, H. Amplitude motion and frequency simulation of a composite viscoelastic microsystem within modified couple stress elasticity. Engineering with Computers 2021, 1-15, https://doi.org/10.1007/s00366-021-01316-8.

28. Khorshidi, M.A. Validation of weakening effect in modified couple stress theory: dispersion analysis of carbon nanotubes. International Journal of Mechanical Sciences 2020, 170, 105358, https://doi.org/10.1016/j.ijmecsci.2019.105358.

29. Al-Furjan, M.S.H.; Samimi-Sohrforozani, E.; Habibi, M., won Jung, D.; Safarpour, H. Vibrational characteristics of a higher-order laminated composite viscoelastic annular microplate via modified couple stress theory. Composite Structures 2021, 257, 113152, https://doi.org/10.1016/j.compstruct.2020.113152.

30. Kwon, Y. R.; Lee, B. C. Numerical evaluation of beam models based on the modified couple stress theory. Mechanics of Advanced Materials and Structures 2020, 1-12, https://doi.org/10.1080/15376494.2020.1825887. 
31. Khan, S.U.; Waqas, H.; Bhatti, M.M.; Imran, M. Bioconvection in the rheology of magnetized couple stress nanofluid featuring activation energy and Wu's slip. Journal of Non-Equilibrium Thermodynamics 2020, 45, 81-95, https://doi.org/10.1515/jnet-2019-0049.

32. Acharya, N.; Mondal, H.; Kundu, P. K. Spectral approach to study the entropy generation of radiative mixed convective couple stress fluid flow over a permeable stretching cylinder. Proceedings of the Institution of Mechanical Engineers, Part C: Journal of Mechanical Engineering Science 2020, 0954406220954893, https://doi.org/10.1177\%2F0954406220954893.

33. Farooq, M.; Khan, A.; Nawaz, R.; Islam, S.; Ayaz, M.; Chu, Y. M. Comparative study of generalized couette flow of couple stress fluid using optimal homotopy asymptotic method and new iterative method. Scientific Reports 2021, 11, 1-20, https://doi.org/10.1038/s41598-021-82746-8.

34. Ellahi, R.; Zeeshan, A.; Hussain, F.; \& Abbas, T. Two-phase couette flow of couple stress fluid with temperature dependent viscosity thermally affected by magnetized moving surface. Symmetry 2019, 11, 647, https://doi.org/10.3390/sym11050647.

35. Arif, M.; Ali, F.; Khan, I.; Nisar, K. S. A time fractional model with non-singular kernel the generalized Couette flow of couple stress nanofluid. IEEE Access 2020, 8, 77378-77395, https://doi.org/10.1109/ACCESS.2020.2982028.

36. Ali, F.; Ahmad, Z.; Arif, M.; Khan, I.; Nisar, K. S. A time fractional model of generalized Couette flow of couple stress nanofluid with heat and mass transfer: applications in engine oil. IEEE Access 2020, 8, 146944146966, https://doi.org/10.1109/ACCESS.2020.3013701.

37. Arif, M.; Ali, F.; Khan, I.; Nisar, K. S. A time fractional model with non-singular kernel the generalized Couette flow of couple stress nanofluid. IEEE Access 2020, 8, 77378-77395, https://doi.org/10.1109/ACCESS.2020.2982028.

38. Prakash, O.; Kumar, D.; Dwivedi, Y. K. MHD free convection flow of a visco-elastic (Kuvshiniski type) dusty gas through a semi infinite plate moving with velocity decreasing exponentially with time and radiative heat transfer. AIP Advances 2011, 1, 022132, https://doi.org/10.1063/1.3599414. 